\title{
Small RNA profiling for identification of microRNAs involved in regulation of seed development and lipid biosynthesis in yellowhorn
}

\author{
Li Wang ${ }^{1,2}$, Chengjiang Ruan ${ }^{1 *}$, Aomin Bao ${ }^{3}$ and $\mathrm{He} \mathrm{Li}^{1}$
}

\begin{abstract}
Background: Yellowhorn (Xanthoceras sorbifolium), an endemic woody oil-bearing tree, has become economically important and is widely cultivated in northern China for bioactive oil production. However, the regulatory mechanisms of seed development and lipid biosynthesis affecting oil production in yellowhorn are still elusive. MicroRNAs (miRNAs) play crucial roles in diverse aspects of biological and metabolic processes in seeds, especially in seed development and lipid metabolism. It is still unknown how the miRNAs regulate the seed development and lipid biosynthesis in yellowhorn.
\end{abstract}

Results: Here, based on investigations of differences in the seed growth tendency and embryo oil content between high-oil-content and low-oil-content lines, we constructed small RNA libraries from yellowhorn embryos at four seed development stages of the two lines and then profiled small RNA expression using high-throughput sequencing. A total of 249 known miRNAs from 46 families and 88 novel miRNAs were identified. Furthermore, by pairwise comparisons among the four seed development stages in each line, we found that 64 miRNAs (53 known and 11 novel miRNAs) were differentially expressed in the two lines. Across the two lines, 15, 11, 10, and 7 differentially expressed miRNAs were detected at 40,54, 68, and 81 days after anthesis, respectively. Bioinformatic analysis was used to predict a total of 2654 target genes for 141 differentially expressed miRNAs (120 known and 21 novel miRNAs). Most of these genes were involved in the fatty acid biosynthetic process, regulation of transcription, nucleus, and response to auxin. Using quantitative real-time PCR and an integrated analysis of miRNA and mRNA expression, miRNA-target regulatory modules that may be involved in yellowhorn seed size, weight, and lipid biosynthesis were identified, such as miR172b-ARF2 (auxin response factor 2), miR7760-p3_1-AGL61 (AGAMOUS-LIKE 61), miR319p_1-FAD2-2 (omega-6 fatty acid desaturase 2-2), miR5647-p3_1-DGAT1 (diacylglycerol acyltransferase 1), and miR7760-p5_1-MED15A (Mediator subunit 15a).

Conclusions: This study provides new insights into the important regulatory roles of miRNAs in the seed development and lipid biosynthesis in yellowhorn. Our results will be valuable for dissecting the post-transcriptional and transcriptional regulation of seed development and lipid biosynthesis, as well as improving yellowhorn in northern China.

Keywords: Yellowhorn, MicroRNA, Lipid biosynthesis, Seed development, Target gene

*Correspondence: ruan@dlnu.edu.cn

${ }^{1}$ Key Laboratory of Biotechnology and Bioresources Utilization, Ministry

of Education, Institute of Plant Resources, Dalian Minzu University,

Dalian 116600, China

Full list of author information is available at the end of the article

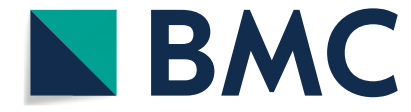

(C) The Author(s) 2021. Open Access This article is licensed under a Creative Commons Attribution 4.0 International License, which permits use, sharing, adaptation, distribution and reproduction in any medium or format, as long as you give appropriate credit to the original author(s) and the source, provide a link to the Creative Commons licence, and indicate if changes were made. The images or other third party material in this article are included in the article's Creative Commons licence, unless indicated otherwise in a credit line to the material. If material is not included in the article's Creative Commons licence and your intended use is not permitted by statutory regulation or exceeds the permitted use, you will need to obtain permission directly from the copyright holder. To view a copy of this licence, visit http://creativecommons.org/licenses/by/4.0/. The Creative Commons Public Domain Dedication waiver (http://creativeco $\mathrm{mmons}$.org/publicdomain/zero/1.0/) applies to the data made available in this article, unless otherwise stated in a credit line to the data. 


\section{Background}

Yellowhorn (Xanthoceras sorbifolium), a member of the Sapindaceae family, is endemic to northern China. This woody oil-bearing species is widely distributed throughout its native range and grows well in barren lands with a dry climate $[1,2]$. Its seed kernels (embryos) can contain as much as $67 \%$ oil content, of which unsaturated fatty acids (FAs) make up to $85-93 \%$. These FAs include linoleic acid (37.1-46.2\%), oleic acid (28.6-37.1\%), and nervonic acid $(1.3-3.1 \%)$ [3, 4], all of which are considered beneficial to human health. Yellowhorn has attracted considerable interest in recent years due to the potential food and medicinal value of its seed oil. However, the seed oil content varies significantly among yellowhorn varieties [5]. To achieve maximum oil yield, a better understanding of the key transcriptional regulatory sites involved in seed development and lipid biosynthesis metabolic pathways is necessary. Investigating the mechanisms of regulation of seed development and lipid biosynthesis in yellowhorn is therefore of practical significance.

Yellowhorn research over the past decade has focused on oil extraction, FA composition, and the use of seed oil as a biodiesel [5-7]. Yellowhorn lipid biosynthesis metabolic pathways have been the subject of several studies, which identified some key genes associated with oil accumulation [8, 9]. For example, two novel diacylglycerol acyltransferase (XsDGAT1 and XsDGAT2) yellowhorn genes were found to control its seed oil content [10]. In addition, de novo transcriptome analysis of multiple yellowhorn tissue types identified lipid biosynthesis and metabolism-related pathways and genes [11]. High- and low-oil yellowhorn embryo tissues at four different developmental stages were analyzed using comparative RNASeq analysis and it was found that many genes played critical roles in promoting oil accumulation, including several transcription factors as well as genes involved in FA biosynthesis, glycolysis/gluconeogenesis, and pyruvate metabolism [12]. The yellowhorn genome sequence was recently sequenced and assembled [13, 14]; besides, based on a large number of single-copy orthologous genes shared by 11 plant genomes, phylogenetic analysis suggested that yellowhorn and longan (Dimocarpus longan) in the Sapindaceae family showed the closest relationship, and diverged from their most recent common ancestor $\sim 33.07$ million years ago [13]. In summary, research focused on the identification of lipid biosynthesis-related genes in yellowhorn has been limited. The regulatory mechanisms involved in seed development and lipid biosynthesis at the post-transcriptional levels (e.g., microRNAs) remain unknown.

MicroRNAs (miRNAs) are small, endogenous, noncoding RNAs produced from stem-loop precursors by
Dicer-catalyzed excisions. MiRNAs direct RNA cleavage or block translation of target transcripts to regulate gene expression post-transcriptionally [15]. A large body of research has shown that miRNAs are critical to diverse aspects of biological and metabolic processes in seeds, including embryogenesis, pattern establishment, and lipid metabolism [16-18]. Zhang et al. [19] found that silencing miR398 in rice can increase panicle length, grain number, and grain size. Thirteen miRNAs were found to regulate oleic acid accumulation in safflower through the deep sequencing of small RNA libraries [20], and 30 miRNAs were found to regulate lipid metabolism in Camelina sativa [21]. Research has determined that many miRNAs control genes that function in FA biosynthesis and accumulation [22, 23]. The miR156 family, for example, directly participates in the regulation of FA biosynthesis in Brassica napus through its targeting of 3-oxoacyl-ACP reductase (KAR) [24], and miR159b, with its targets omega-6 fatty acid desaturase 2 (FAD2), fatty acid elongase 1 (FAE1), and fatty acyl-ACP thioesterase $B(F A T B)$, can affect the levels of oleic acid, palmitic acid, and eicosenoic acid in Arabidopsis thaliana seeds [25]. In addition, the regulatory module miR167A-ARF8 (auxin response factor 8 ) has been found to affect the $\alpha$-linolenic acid content and seed size in C. sativa [26]. Taken together, this body of evidence indicates that miRNAs have diverse biological functions related to seed development and lipid biosynthesis in many plant species. However, the biological information of miRNAs of yellowhorn is currently limited, and this research remains in the initial stage. Previous reports have mainly focused on the effects of miRNA on floral organ development and sex differentiation [27-29]. Even so, the miRNA-mediated regulatory networks that determine seed development and oil accumulation are poorly understood in the woody oil crop yellowhorn.

In the present study, yellowhorn embryos at four seed developmental stages $(40,54,68$, and 81 days after anthesis (daa) from high-oil-content $(\mathrm{HO})$ and low-oilcontent (LO) lines were used to construct small RNA (sRNA) libraries. The miRNA expression was then profiled using high-throughput sequencing. In total, 249 known miRNAs and 88 novel miRNAs were identified in yellowhorn. Computational analysis was used to detect differentially expressed miRNAs among different developmental stages and among embryos from highand low-oil lines. In addition, an association analysis between miRNA and mRNA expression was conducted to elucidate the regulatory relationship between miRNAs and their corresponding mRNA targets. This analysis was studied to understand seed development and lipid biosynthesis in yellowhorn. 


\section{Results}

\section{Changes in the oil content, seed size, and weight at different seed developmental stages}

The embryo oil content, seed size, and weight were examined at four seed developmental stages $(40,54$, 68 , and 81 daa) in the $\mathrm{HO}$ and LO lines. Images of yellowhorn seeds and embryos were produced at four seed developmental stages for comparison (Fig. 1a).
Both lines showed a rapid accumulation of the embryo oil content from 40 to 68 daa followed by a very slow increase from 68 to 81 daa; the highest oil content was produced at 81 daa (Fig. 1b). In both yellowhorn lines, the oil content accumulated at the highest rate between 40 and 54 daa, indicating that substantial lipid biosynthesis mainly occurred during the early to middle stages of seed development. There was significantly

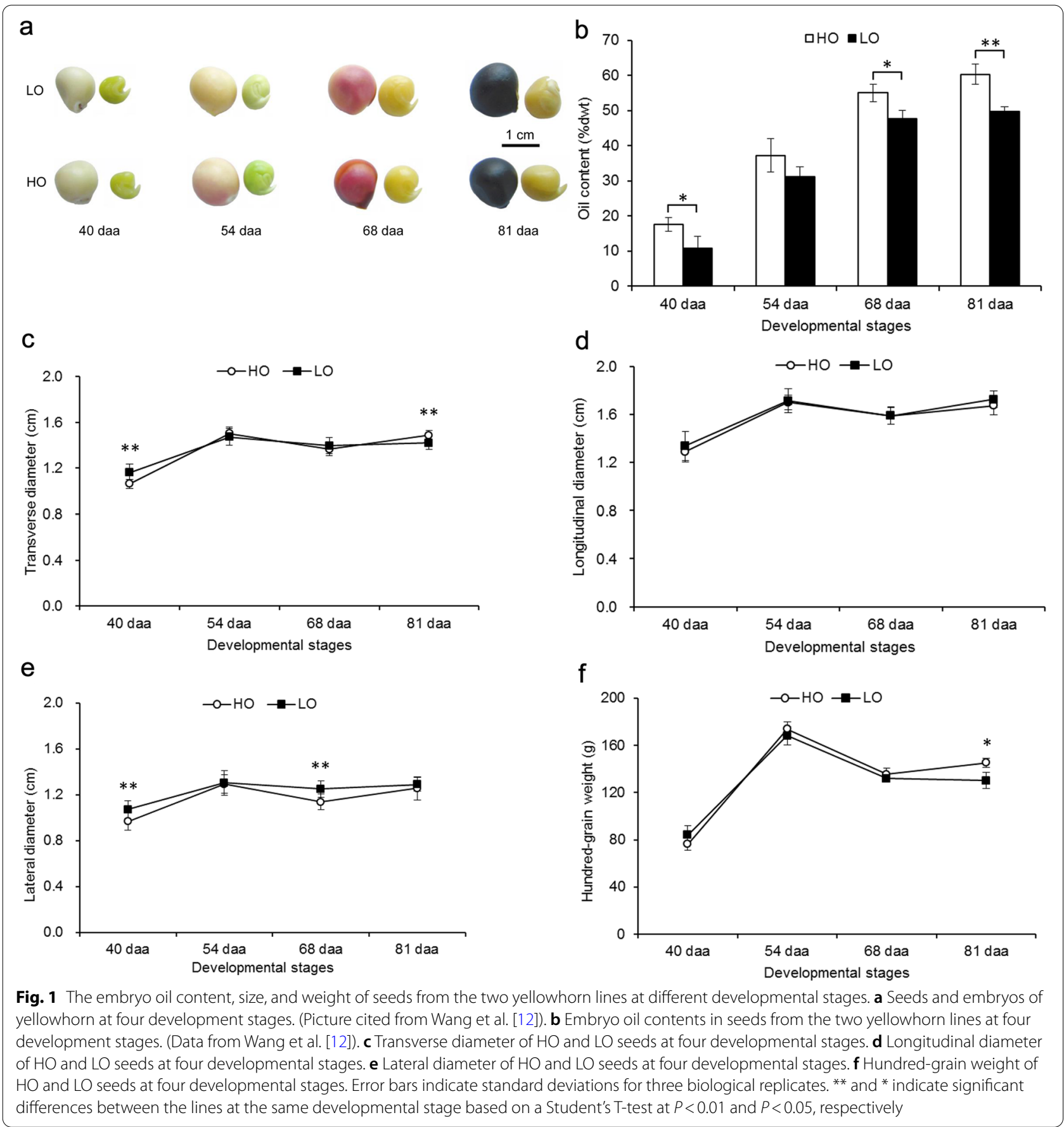


higher oil content in the $\mathrm{HO}$ embryos than in the $\mathrm{LO}$ embryos at 40, 68, and 81 daa, but not at 54 daa. At 81 daa, the oil content of $\mathrm{HO}$ embryos was $10.69 \%$ higher than that of LO (Fig. 1b).

Additionally, the transverse diameters, longitudinal diameters, and lateral diameters of the seeds increased in both lines from 40 to 54 daa and then decreased at 68 daa followed by a slow increase from 68 to 81 daa, indicating that faster growth of developing seeds occurred mainly at the early stages (40-54 daa) (Fig. 1c-e). At 54 daa, the seed sizes of the two lines were at the highest level. Moreover, the transverse diameters and lateral diameters of the seeds from the HO line were significantly smaller than those from the LO line at 40 daa, and the lateral diameters of the seeds from the $\mathrm{HO}$ line were significantly smaller than those from the LO line at 68 daa (Fig. 1c, e). With seed development, the change in the weight of the two lines was similar to the change of the seed size, and the HO line had a higher hundred-grain weight than the LO line (Fig. 1f).

\section{Overview of sRNA sequencing in yellowhorn}

Sixteen sRNA libraries from eight samples (two biological replicates for each) were constructed to identify miRNAs linked to the regulation of oil accumulation and seed development. A total of 238,325,077 raw reads were obtained from these libraries by high-throughput sequencing (approximately 14.9 million raw reads per library). A total of $194,958,060$ clean reads of $18-25$ nucleotides (nt) and an average of 12,184,879 reads $(81.80 \%)$ per library were obtained after removing the adaptor dimers, junk reads, low-complexity sequences, and sequences shorter than 18 nucleotides and longer than 25 nucleotides (Table 1).

Following a search against the Rfam and Repbase databases for sRNA sequences by the Bowtie software [30], an average of 3.22 and $7.17 \%$ clean reads in the $\mathrm{HO}$ and LO lines, respectively, were mapped to known non-coding sRNAs (rRNAs, tRNAs, snRNAs, snoRNAs, and other Rfam RNAs) and repeat sequences (Additional file 1: Table S1; Additional file 2: Table S2). Moreover, an average of 5.69 and $4.41 \%$ clean reads in the $\mathrm{HO}$ and LO lines, respectively, were mapped to NATs (Natural Antisense Transcripts) and phased siRNAs (phasiRNAs). Notably, about $5 \%$ of the clean reads per library, on average, were mapped to miRNAs in each line (Additional file 1: Table S1; Additional file 2: Table S2). The proportion of miRNAs declined at 81 daa compared with 40,54, and 68 daa in the two lines, implying that a body of miRNAs were induced in the early and middle stages of seed development. The length distribution of unique sRNAs in both lines at four developmental stages were then summarized. Most sRNA reads ranged from 21 to $24 \mathrm{nt}$ in length, leading to similar length distributions in both lines at the different developmental stages. Twenty-four nt sRNAs were the most abundant, accounting for $30.74 \%$ (LO81) to $75.95 \%$ (HO40) of the total (Fig. 2). Also

Table 1 Summary of small RNA sequencing statistics

\begin{tabular}{|c|c|c|c|c|c|}
\hline Sample & & Raw reads & $\begin{array}{l}\text { Adaptor sequence and length with } \\
<18 \mathrm{nt} \text { and }>25 \mathrm{nt}\end{array}$ & Junk reads & Clean reads \\
\hline \multirow[t]{2}{*}{$\mathrm{HO} 40$} & HO40_1 & $17,087,566$ & $1,824,397$ & 160,613 & $15,102,556$ \\
\hline & HO40_2 & $15,381,430$ & $1,191,349$ & 131,834 & $14,058,247$ \\
\hline \multirow[t]{2}{*}{$\mathrm{HO} 54$} & HO54_1 & $15,504,184$ & $1,464,666$ & 147,468 & $13,892,050$ \\
\hline & HO54_2 & $18,792,450$ & $2,046,587$ & 134,962 & $16,610,901$ \\
\hline \multirow[t]{2}{*}{ HO68 } & HO68_1 & $12,770,282$ & $1,373,111$ & 82,560 & $11,314,611$ \\
\hline & HO68_2 & $13,660,269$ & $4,816,480$ & 30,860 & $8,812,929$ \\
\hline \multirow[t]{2}{*}{$\mathrm{HO} 81$} & HO81_1 & $13,489,436$ & $2,151,260$ & 62,326 & $11,275,850$ \\
\hline & HO81_2 & $10,989,687$ & $2,342,466$ & 74,391 & $8,572,830$ \\
\hline \multirow[t]{2}{*}{ LO40 } & LO40_1 & $14,051,494$ & $2,970,987$ & 122,646 & $10,957,861$ \\
\hline & LO40_2 & $13,797,449$ & $4,423,308$ & 93,188 & $9,280,953$ \\
\hline \multirow[t]{2}{*}{ LO54 } & LO54_1 & $14,790,646$ & $3,030,592$ & 54,704 & $11,705,350$ \\
\hline & LO54_2 & $14,346,000$ & $2,721,911$ & 108,500 & $11,515,589$ \\
\hline \multirow[t]{2}{*}{ LO68 } & LO68_1 & $13,911,458$ & $1,846,479$ & 82,175 & $11,982,804$ \\
\hline & L068_2 & $16,195,799$ & $1,553,201$ & 79,763 & $14,562,835$ \\
\hline \multirow[t]{2}{*}{ LO81 } & L081_1 & $17,910,804$ & $2,992,808$ & 74,723 & $14,843,273$ \\
\hline & L081_2 & $15,646,123$ & $5,115,030$ & 61,672 & $10,469,421$ \\
\hline Total & & $238,325,077$ & $41,864,632$ & $1,502,385$ & $194,958,060$ \\
\hline Average & & $14,895,317$ & $2,616,540$ & 93,899 & $12,184,879$ \\
\hline
\end{tabular}


common were 21,22 , and 23 nt sRNAs, which were more abundant than those of any other length besides $24 \mathrm{nt}$.

\section{Identification of known and novel miRNAs}

A total of 249 known miRNAs were identified in the 16 libraries by analyzing the alignment results against the miRbase 21.0 database (Additional file 3: Table S3). Among the identified known miRNA sequences, 180 were identified to belong to 46 miRNA families. And the miRNA sequences were mapped to known miRNAs from 34 other plant species, with the highest number of miRNAs mapped to known mtr-miRNAs of Medicago truncatula (54, 21.67\%), followed by gma-miRNAs of Glycine max (39, 15.66\%), ptc-miRNAs of Populus trichocarpa (22, 8.84\%), and osa-miRNAs of Oryza sativa (17, 6.83\%) (Fig. 3; Additional file 4: Table S4). Among the identified miRNA families, the MIR159 family had the largest number of members (18), followed by the MIR171_1 and MIR482 families with 14 members, and MIR156 and MIR166, with 12 and 10 members, respectively (Fig. 4). The expression profiles of the known miRNAs showed that the normalized read counts of known miRNAs varied from 100,000 to less than 10 reads, exhibiting great variation, even within the same family (Additional file 3: Table S3). At the opposite end of the

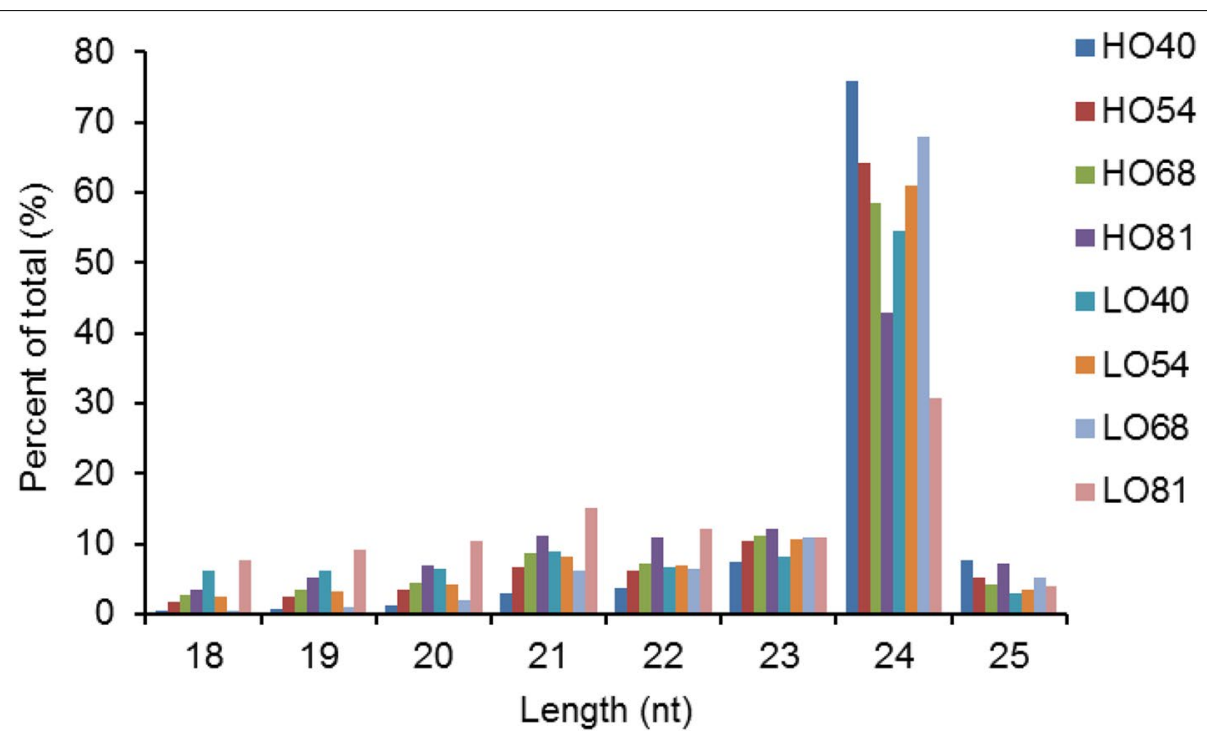

Fig. 2 Length distribution of unique sRNAs in two yellowhorn lines at four developmental stages

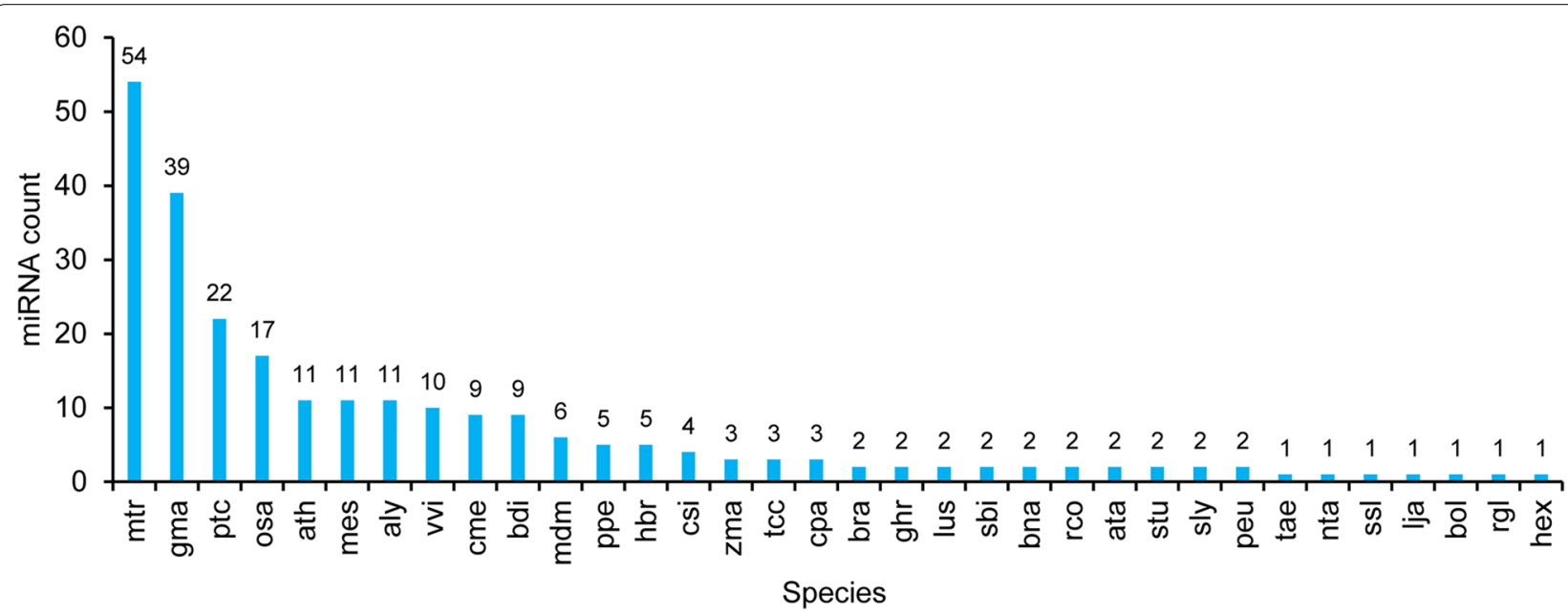

Fig. 3 Species distribution of known miRNAs identified in yellowhorn 

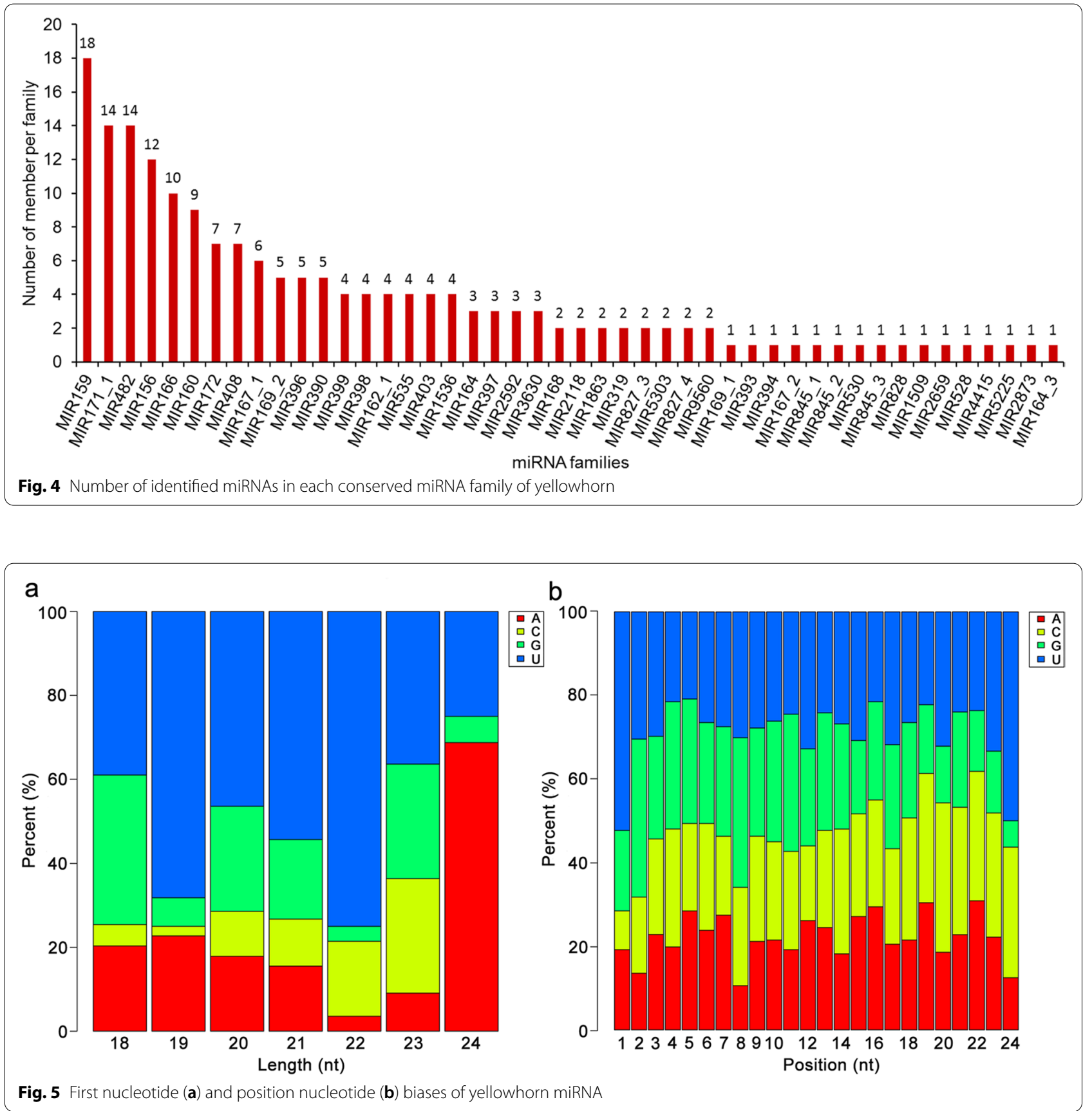

spectrum, about 49 miRNAs were identified that had fewer than 10 reads in all libraries. In addition, Fig. 5a shows the distribution of miRNA first nucleotide preferences. MiRNAs of $24 \mathrm{nt}$ tended to begin with $5^{\prime}$-A, while 21-22 nt miRNAs tended to start with $5^{\prime}-\mathrm{U}$. At the $5^{\prime}$ end, uridine was the most common nucleotide $(>52 \%)$ for all known miRNAs (Fig. 5b).

In addition to the known miRNAs, the remaining unaligned sRNA sequences were mapped to the yellowhorn genome [31] and their hairpin structures were predicted to identify novel miRNAs in yellowhorn. We only considered sRNAs that exhibited the typical stem-loop structure. The novel miRNA sequences were named in the form of Xso-miRn-number. A total of 88 novel miRNAs between $18 \mathrm{nt}$ to $25 \mathrm{nt}$ in length were identified. Among them, $61.36 \%$ were $24 \mathrm{nt}$ in length (Additional file 5: Table S5). The novel miRNA precursors ranged in length from $64 \mathrm{nt}$ to $202 \mathrm{nt}$, with an average 
length of $140 \mathrm{nt}$. Compared to known miRNAs, most of the identified novel miRNAs had relatively low read counts (normalized). Most of the novel miRNAs had fewer than 10 reads, and only two novel miRNAs had more than 100 reads in all libraries (Additional file 5: Table S5). The minimum free energies of the hairpin structures of these miRNA precursors ranged from $-130.1 \mathrm{kcal} \cdot \mathrm{mol}^{-1}$ to $-26.9 \mathrm{kcal} \cdot \mathrm{mol}^{-1}$, with an average of $-59.6 \mathrm{kcal} \cdot \mathrm{mol}^{-1}$, similar to those of other plant miRNA precursors [32]. The secondary structures of the four most abundant novel miRNAs (Xso-miRn24, XsomiRn44, Xso-miRn45, and Xso-miRn84) were predicted (Fig. 6), indicating they can form typical stem-loop hairpins, and their folding free energies were $-53.4,-60.9$, -60.9 , and $-60.8 \mathrm{kcal} \cdot \mathrm{mol}^{-1}$, respectively. Additionally, to evaluate the consistency of the biological replicates, Principal Components Analysis (PCA) was performed on the 16 samples using the expression profiles of all known and novel miRNAs. As shown in Fig. 7, every two replicates were relatively clustered together, indicating the reliability as well as operational stability of the experimental results.

\section{Differential expression of miRNAs in embryos during seed development}

Pairwise comparison between different developmental stages in each line was conducted using the normalized expression levels of miRNAs to identify differentially expressed miRNAs $(P$-values $<0.05)$. In total, 53 known miRNAs and 11 novel miRNAs were found to be differentially expressed in pairwise comparisons (HO40 vs. HO54, HO54 vs. HO68, HO68 vs. HO81, LO40 vs. LO54, LO54 vs. LO68, and LO68 vs. LO81). Among them, only five known miRNAs (miR390, miR156i-3p_1, miR482b3p_1, miR166a_1, and miR156e-3p_1) expressed at high levels (Additional file 6: Table S6; Additional file 7: Table S7).

In the HO line, 11 miRNAs were significantly differentially expressed between 40 and 54 daa (HO40 vs. HO54), including 5 and 6 miRNAs with upregulated and downregulated expression in $\mathrm{HO} 54$, respectively (Additional file 6: Table S6a). And 8 differentially expressed miRNAs were identified between 54 and 68 daa (HO54 vs. HO68), which included 3 upregulated miRNAs and 5 downregulated miRNAs in HO68 (Additional file 6: Table S6b). Furthermore, four miRNAs were upregulated and another four miRNAs were downregulated at the fully mature stage (81 daa) of the HO line compared with 68 daa (Additional file 6: Table S6c). In the LO line, there were 17 significantly differentially expressed miRNAs between 40 and 54 daa (LO40 vs. LO54). When compared to LO40, 6 and 11
miRNAs were upregulated and downregulated in LO54, respectively (Additional file 7: Table S7a). In the LO54 vs. LO68 comparison, 7 miRNAs were upregulated and 8 miRNAs were downregulated in LO68 (Additional file 7: Table S7b). Moreover, 20 miRNAs showed significant differential expression between 68 and 81 daa (LO68 vs. LO81), of which 10 miRNAs were upregulated and another $10 \mathrm{miRNAs}$ were downregulated in LO81 (Additional file 7: Table S7c). Additionally, we found two differential miRNAs (upregulated miR156ep3_1 and downregulated miR172b) in both lines at 40 vs. 54 daa and 54 vs. 68 daa, respectively.

\section{Differentially expressed miRNAs}

between the high-oil-content and low-oil-content lines

The differentially expressed miRNAs between the two lines were compared. The significantly differentially expressed miRNAs between two samples with a $P$-value $<0.05$ are presented in Fig. 8. At 40 daa, 5 miRNAs were significantly upregulated and 10 miRNAs (e.g., miR156e-3p_1 and miR172b) were downregulated in the $\mathrm{HO}$ line compared with the LO line. At 54 daa, six miRNAs were upregulated in the $\mathrm{HO}$ line, while the other five miRNAs (miR172b, miR390_1, miR397-5p, miR172c-5p_1, and miR156e-p3_1) were downregulated in the $\mathrm{HO}$ line compared with the LO line. At 68 daa, six upregulated miRNAs (e.g., miR171i-p5_1) were found in the $\mathrm{HO}$ line, whereas the other four miRNAs (miR169a-3p_1, miR172b, Xso-miRn62, and Xso-miRn27) were preferentially expressed in the LO line. We also identified seven downregulated miRNAs (e.g., miR319p_1, miR5655-p3, and miR7760-p3_1) between fully mature embryos of the $\mathrm{HO}$ and LO lines. Interestingly, one co-expressed differential miRNA (miR172b) was detected that was downregulated at the early and middle stages of seed development (40, 54 , and 68 daa) in the $\mathrm{HO}$ line relative to the LO line at the corresponding developmental stages. This indicated that this known miRNA might play an important role in the early and middle stages of yellowhorn seed development.

\section{Identification of differentially expressed miRNA target genes and gene enrichment analysis}

Target mRNA is regulated by miRNA through translational repression or mRNA degradation. A total of 141 differentially expressed miRNAs (120 known and 21 novel miRNAs) were putatively targeted to 2654 genes to identify any correlation between the expression of differentially expressed miRNAs and mRNAs (Additional file 8: Table S8). Some miRNA target genes were annotated as transcription factor-coding genes, 


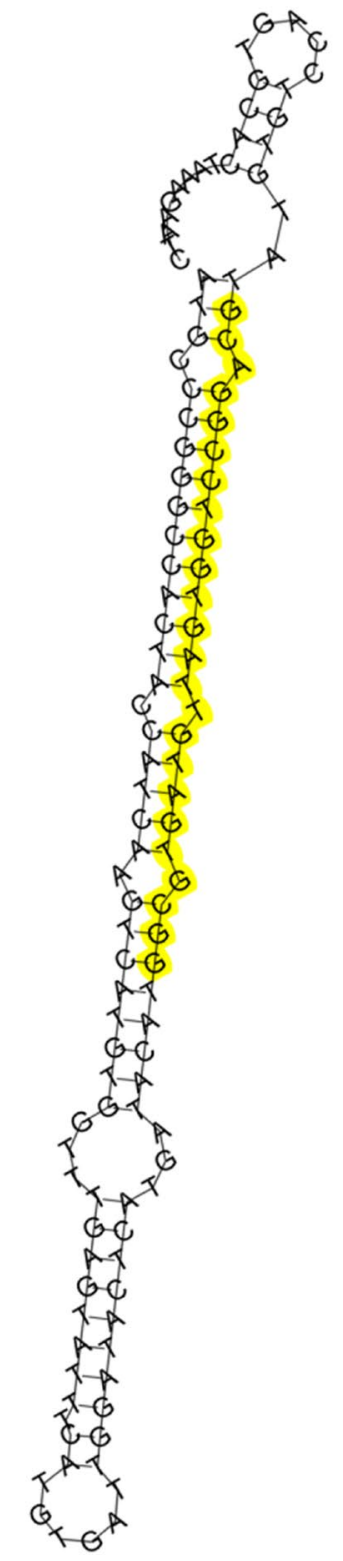

Xso-miRn24

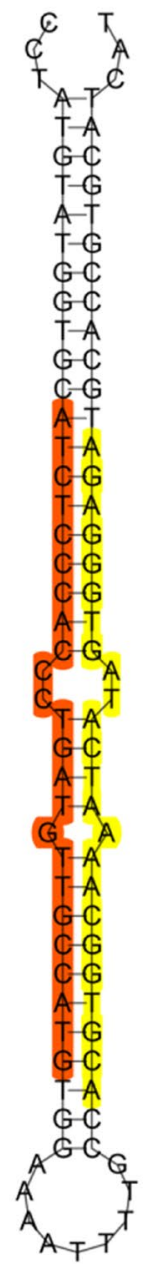

Xso-miRn44/Xso-miRn45

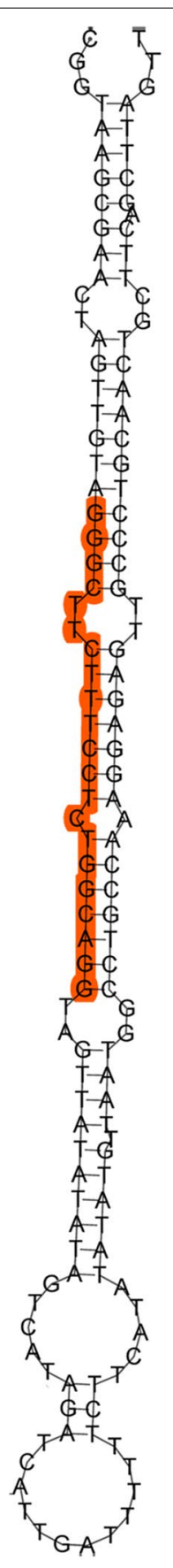

Xso-miRn84

Fig. 6 Secondary structures of the four most abundant novel miRNA precursors. Red (5p) and yellow (3p) indicate segments corresponding to the mature miRNAs

including ARF2, which was targeted by miR172b; growth-regulating factor 5 (GRF5), which was targeted by miR171i-p5_1; ethylene-responsive transcription factor 3 (ERF3), which was targeted by miR171k-5p_1; MADS domain protein AGAMOUS-LIKE 61 (AGL61), which was targeted by miR7760-p3_1; and WRKY transcription factor 41 (WRKY41), which was targeted by Xso-miRn80 (Additional file 9: Table S9). MiR7760-p3_1 was also 


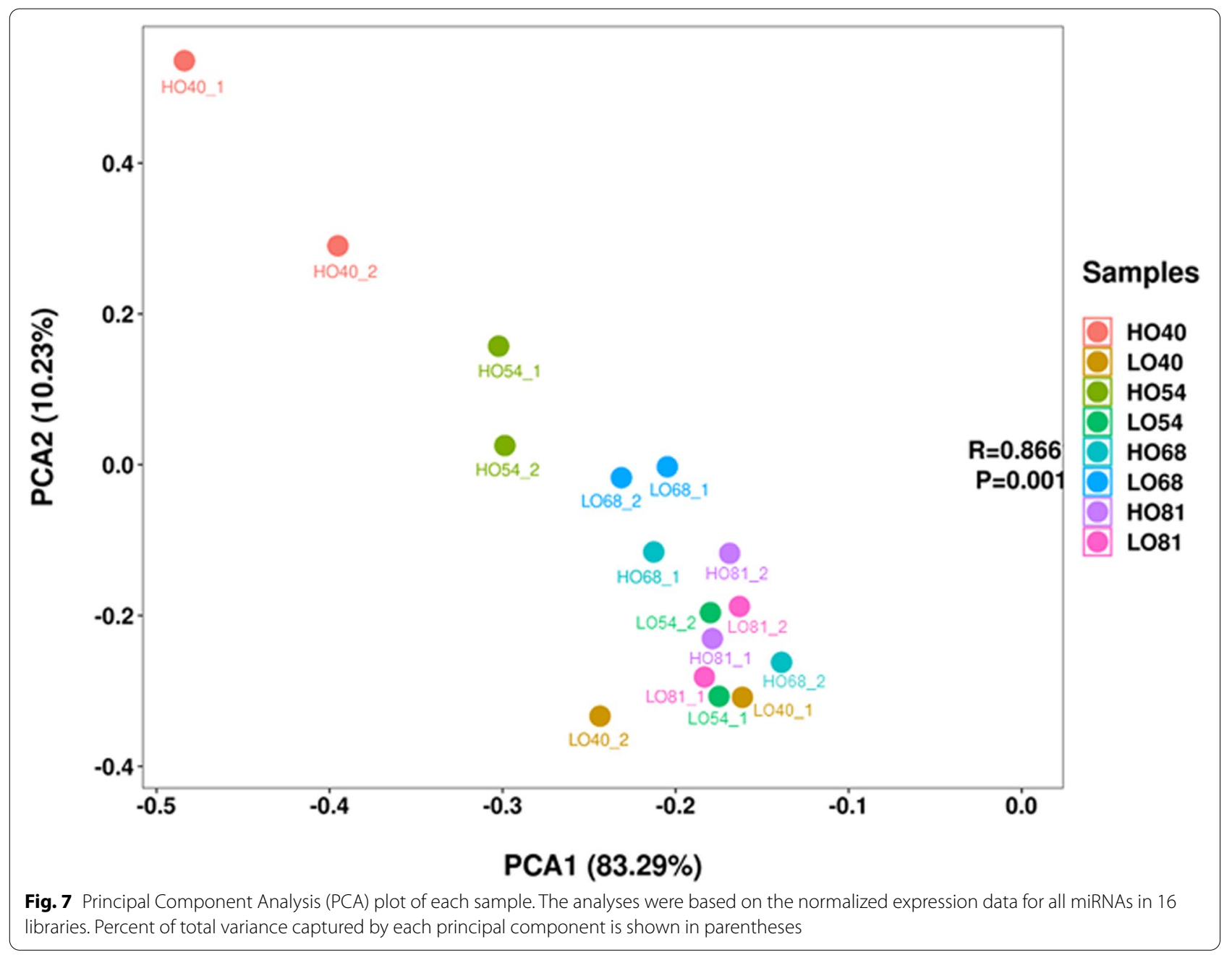

predicted to target the KAR gene; miR319p_1 targets the FAD2-2 gene; miR1536-p5_2 targets the lysophosphatidyl acyltransferase 5 (LPAT5) gene; miR5647-p3_1 targets the DGAT1 gene and miR7760-p5_1 was predicted to target the Mediator subunit 15a (MED15A) gene (Additional file 9: Table S9). These miRNA-target regulatory modules are reported here in yellowhorn for the first time.
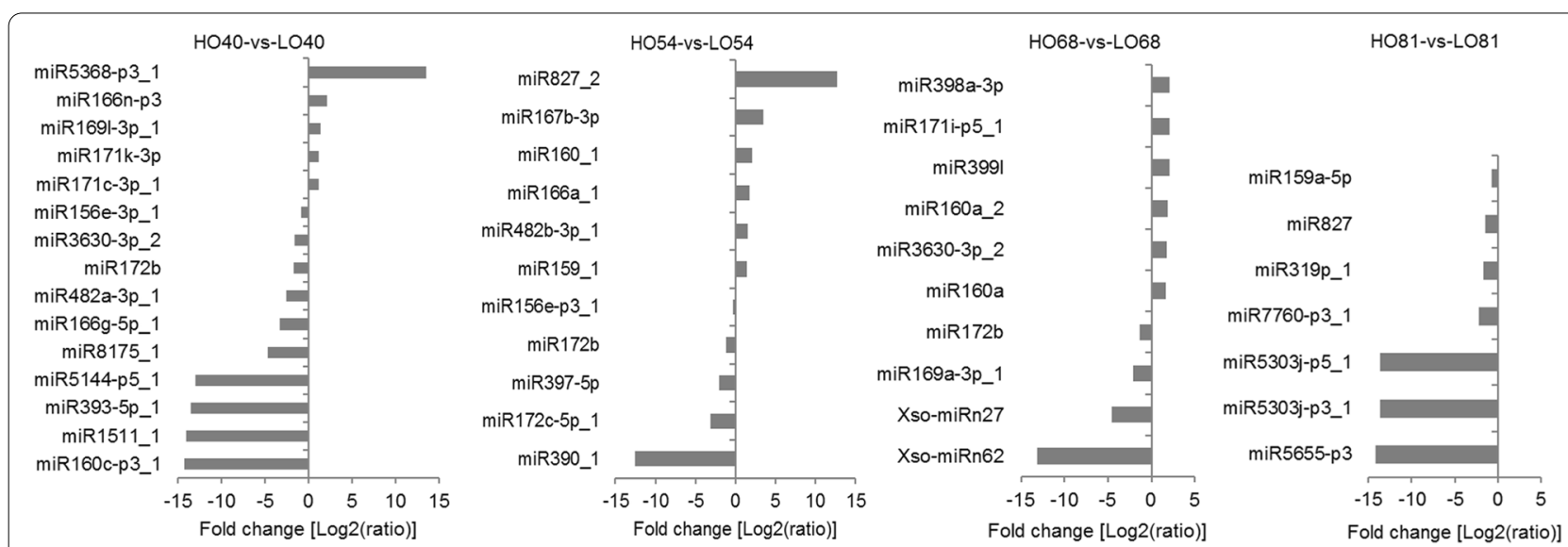

Fig. 8 Differentially expressed miRNAs between the $\mathrm{HO}$ and $\mathrm{LO}$ lines during seed development 


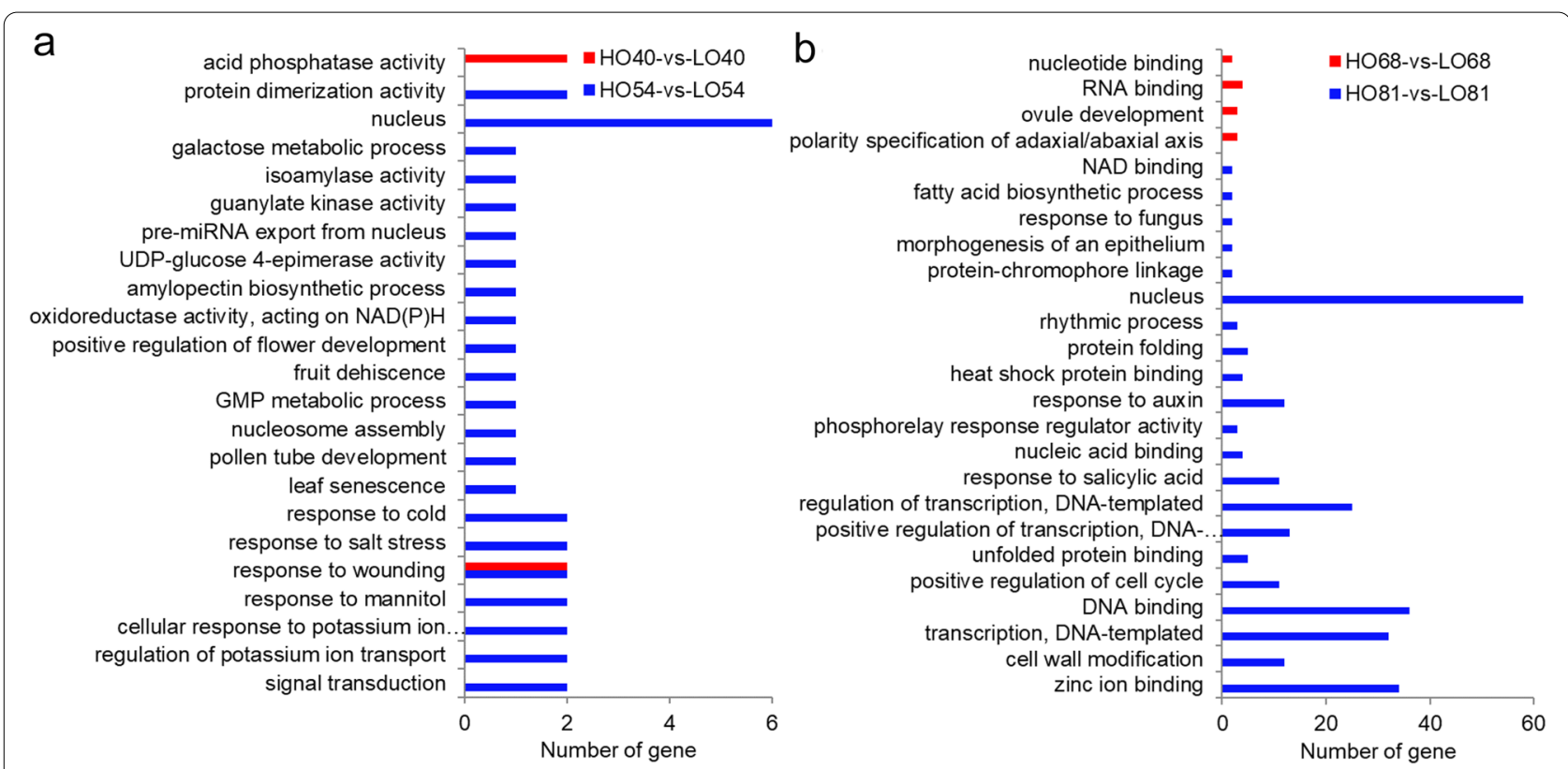

Fig. 9 Gene Ontology categories and sub-categories of the differentially expressed miRNA targets between the following samples: a HO40 vs. LO40 and HO54 vs. LO54, and b HO68 vs. LO68 and HO81 vs. LO81

The differentially expressed miRNA targets between two lines were subjected to Gene Ontology (GO) enrichment analysis. One oil accumulation-related GO term (FA biosynthetic process (GO:0006633) was found to be significantly enriched by conducting GO annotation of these target genes. Notably, the most enriched GO terms were the nucleus, DNA binding, transcription, regulation of transcription, positive regulation of transcription, zinc ion binding, response to auxin, and cell wall modification (Fig. 9). The target genes of the differentially expressed miRNAs (miR7760-p3_1) in HO81 and LO81 were involved in the regulation of FA biosynthetic process, positive regulation of transcription, zinc ion binding, and NAD binding (Additional file 8: Table S8). The functions of the predicted target genes were diverse, suggesting that these differentially expressed miRNAs may play important roles during seed development and lipid biosynthesis in yellowhorn.

Kyoto Encyclopedia of Genes and Genomes (KEGG) pathway analysis of all differentially expressed miRNA targets showed that 268 target genes targeted by 52 of differentially expressed miRNAs mapped to 145 pathways (Additional file 10: Table S10), including those correlated with amino acid metabolism and lipid biosynthesis and metabolism, such as histidine metabolism, biosynthesis of unsaturated fatty acids, fatty acid biosynthesis, glycosphingolipid biosynthesis-ganglio series, glycerolipid metabolism, and fatty acid metabolism. Notably, the miRNAs miR319p_1, miR7760-p3_1, miR7760-p5_1, and miR5655-p3 were associated with biosynthesis of unsaturated fatty acids and fatty acid biosynthesis through targeting the FAD2-2, KAR, short-chain type dehydrogenase/reductase (SDR1), and pentatricopeptide repeat-containing protein (PCMP-H42) genes, respectively (Additional file 9: Table S9; Additional file 10: Table S10).

\section{Features and the evolutionary conservation of the target gene encoding MED15A}

With respect to the target gene (cDNA sequence) encoding MED15A, which was designated as XsM$E D 15 A$, it had 3467 nucleotide residues containing a complete 2463-bp open reading frame (ORF), which was predicted to code for 820 amino acids-polypeptide with a deduced molecular weight of $89.02 \mathrm{kDa}$ and isoelectric point of 8.08 (Additional file 11: Fig. S1). XsMED15A amino acid sequence was submitted online and the results showed that it contained a coiled coil region and two internal repeats, without signal peptide. Multiple sequence alignments indicated that XsMED15A mature peptide was highly conserved with Mediator subunit 15a found in other plants. It shared $81 \%$ identity with the MED15A protein of Pistacia vera (XP_031251638.1), 80\% with Citrus clementina (XP_024036133.1), 78\% with Juglans regia (XP_035548095.1), 77\% with Malus domestica (XP_028955279.1), 76\% with Gossypium 
hirsutum (XP_040969330.1), and 74\% with G. max (XP_003547623.1) (Additional file 12: Fig. S2).

\section{Quantitative real-time PCR validation of miRNA and corresponding target genes}

To validate the expression patterns of the miRNAs derived from the high-throughput sequencing, five miRNAs (miR172b, miR171i-p5_1, miR7760-p3_1, miR319p_1, and Xso-miRn80) and nine target genes (ARF2, GRF5, AGL61, KAR, FAD2-2, WRKY41, LPAT5, DGAT1, and MED15A) were selected and qRTPCR analysis was performed. The resulting qRT-PCR data for the differentially expressed miRNAs were nearly consistent with the sequencing results, and the expression changes of the target genes showed an inverse correlation with differentially expressed miRNAs (Fig. 10). MiR7760-p3_1, however, showed relatively low expression in both lines at 40-68 daa compared with 81 daa, and showed exclusive expression at 81 daa in the sequencing data.

\section{Discussion}

Complex sRNA populations involved in seed development and lipid biosynthesis of yellowhorn

Yellowhorn is an endemic and economically important oil-rich tree in northern China that can withstand cold and drought conditions [13]. The oil production of oil

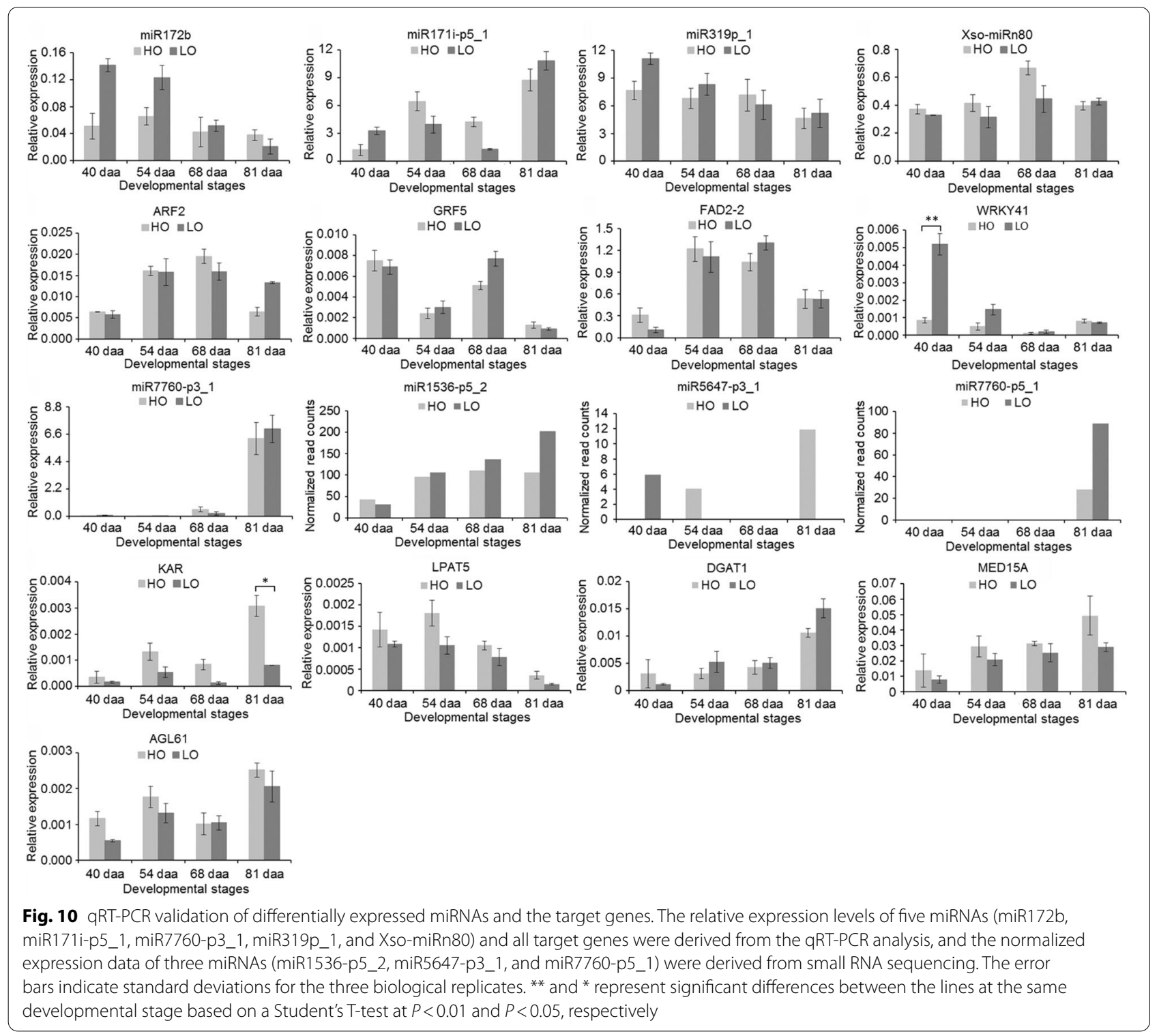


plant seeds is mainly determined by the seed size, seed weight, and embryo oil content. However, the complex regulatory mechanisms of seed development and oil biosynthesis affecting oil production in yellowhorn are still poorly understood. In this study, the dynamic growth patterns (size and weight) and the embryo oil content of developing seeds in $\mathrm{HO}$ and $\mathrm{LO}$ yellowhorn lines were analyzed, and faster growth and higher oil accumulation in developing seeds were detected at 40-54 daa and 40-68 daa, respectively (Fig. 1). Furthermore, the HO line had a higher embryo oil content, higher hundredgrain weight, and smaller seeds than the LO line (Fig. 1). Though these $\mathrm{HO}$ and LO lines shared similar genetic backgrounds, they exhibited large phenotypic differences, which are of interest in terms of attempts to understand their underlying molecular mechanisms.

MiRNAs as systemic regulators are involved in plant seed development and lipid metabolism [17, 25, 33]. Although some functional genes responsible for lipid biosynthesis in developing yellowhorn seeds were cloned and identified in previous studies $[9,10,12]$, the role of miRNAs in seed developmental processes, for example, seed size, and lipid biosynthesis, remains unknown. In this study, we constructed sRNA populations of the $\mathrm{HO}$ and LO lines at four seed developmental stages. The 16 sRNA libraries had abundant high-quality data. From the yellowhorn embryo sRNA library, 249 known miRNAs belonging to 46 families were identified, and conserved across 34 plant species, including notable conservation with $M$. truncatula, G. max, and P. trichocarpa; this suggested a close relationship between these plant species and yellowhorn, nearly in accordance with the report by Wang et al [29]. Some known miRNAs from 9 miRNA families (e.g., MIR156, MIR159, MIR168, MIR166, and MIR482) demonstrated high expression levels in all libraries, validating their broad evolutionary conservation. In addition, 88 novel miRNAs were identified, most of which showed much lower expression levels than the known miRNAs. Only two novel miRNAs (Xso-miRn42 and Xso-miRn44) exhibited relatively higher expression levels (normalized read counts $>100$ ) in all libraries.. These findings suggested that a complex, diverse array of sRNAs were involved in yellowhorn seed development and lipid biosynthesis.

\section{MiRNAs and their target genes associated with the seed development}

Auxin is implicated in various physiological and developmental processes in plants [34]. Many investigations indicated that auxin response factors regulated plant seed development by controlling auxin responses [33]. For example, abnormal embryo symmetry (tri- or quadrilateral instead of bilateral cotyledons) defects occurred in plants that expressed miR160-resistant ARF17 [35]. ARF2 is linked to seed size in plants [36]. In Arabidopsis, the megaintegumenta (mnt) mutant, which has a lesion in the $A R F 2$ gene, was found to produce larger seeds than wild-type Arabidopsis [37]. In the present study, miR172b was predicted to target the $A R F 2$ gene. The ARF2 gene showed slightly higher expression in $\mathrm{HO}$ than in LO at 40-68 daa, with an exception occurring at 81 daa (Fig. 10). In contrast, miR172b was found to be downregulated during seed development in $\mathrm{HO}$ compared to $\mathrm{LO}$ in the early to middle stages (Figs. 8 and 10). These data combined with the finding that the $\mathrm{HO}$ line exhibited smaller seeds compared to the LO line (Fig. 1c-e) suggested that miR172b might positively regulate seed size in yellowhorn through the suppression of the $A R F 2$ gene. Previous studies showed that the miR172 family positively affected seed weight through the suppression of APETALA2-like transcription factors in Arabidopsis [38, 39]; thus, we presume that the members of the miR172 family have diverse biological functions in the seed development of plants.

The growth-regulating factor family is composed of plant-specific transcription factors that play various roles in growth and development, such as in seed formation [40, 41] and leaf development [42]. In A. thaliana, AtGRF5 participated in the positive control of cell proliferation during leaf development [43]. Owing to an increased cell number, AtGRF5 overexpression lines had larger leaf areas, while the atgrf5 single-gene mutant had narrower leaves compared to the wild type due to a decreased cell number [43]. Furthermore, GRF5overexpressing lines had improved photosynthetic performance, increased chloroplast number, and higher tolerance to nitrogen depletion compared with wild-type plants [44]. A large body of research has determined that miR396 directly regulates the expression of some GRFs at the post-transcription level [16, 45]. However, AtGRF5 has no miR396 target site and is therefore not regulated by this miRNA in A. thaliana [46, 47]. Notably, miR171i-p5_1 targets the GRF5 gene in yellowhorn seeds. MiR171i-p5_1 exhibited decreased expression from 54 to 68 daa, followed by a sharp increase at 81 daa in the two lines, while GRF5, its target gene, exhibited a trend opposite to that of miRNA (Fig. 10). Additionally, the expression level of GRF5 was lower in HO than in LO at 54 and 68 daa, whereas miR171i-p5_1 was more highly expressed in $\mathrm{HO}$ compared to $\mathrm{LO}$ at the corresponding developmental stages and was particularly significantly upregulated at 68 daa (Figs. 8 and 10). These results suggested that the GRF5 gene is negatively regulated by miR171i-p5_1 in yellowhorn, and smaller seeds in the $\mathrm{HO}$ line may be attributed to a negative regulatory role of miR171i-p5_1. 
MADS-box genes are critical in plant development, especially in gamete and seed development. The MADS domain protein AGL62 regulates the timing of endosperm cellularization [48]. Recently, research has shown that OsMADS87 plays a role in endosperm cellularization initiation and in the regulation of the final seed formation in rice [49]. AGL61 was also found to be expressed in the central and endosperm cells. In the agl61 mutant central cell development was impaired, resulting in a maternal-lethal phenotype [50]. In this study, we found that the AGL61 gene, targeted by miR7760-p3_1, showed higher expression in $\mathrm{HO}$ than in $\mathrm{LO}$ at 40,54 , and 81 daa (Fig. 10). As expected, miR7760-p3_1 showed lower expression in $\mathrm{HO}$ compared to $\mathrm{LO}$ at 40,54, and 81 daa and was particularly significantly downregulated in $\mathrm{HO}$ at 81 daa (Figs. 8 and 10), indicating that miR7760p3_1 mainly functions in the mature stage of seed development by negatively regulating the expression of AGL61. In a previous study, it was shown that after pollination, the yellowhorn embryo sac gradually accumulated a high liquid content, including free nuclear endosperm, a small amount of cellular endosperm, and other soluble material [51]. In addition, when the ovule develops into a mature seed, the endosperm is completely absorbed by the embryo. Therefore, the increase of seed weight in the HO line compared to LO may be the result of an increase in seed filling during the maturation phase due to greater absorption of the endosperm caused by higher AGL61 expression. Further work is clearly required to completely understand the roles and involvement of AGL61 in yellowhorn seed development.

\section{MiRNAs and their targets are related to yellowhorn lipid biosynthesis}

The sequencing of oil crops has yielded many miRNAs associated with lipid metabolism-related genes in recent years. For example, 97, 40, 30, and $4 \mathrm{miR}$ NAs targeting $89,15,133$, and 4 lipid biosynthesis genes were reported for soybean [52], B. napus [24], C. sativa [21], and peanut [22], respectively. Here, we detected five differentially expressed miRNAs targeting five candidate genes regulating lipid biosynthesis functions during seed development. Among these miRNAs, miR7760-p3_1 regulates $K A R$, which is directly involved in fatty acid biosynthesis $[11,53]$, as well as AGL61, which is involved in seed development. In contrast to miR7760-p3_1, the KAR gene, which encodes 3-ketoacyl-ACP reductase and catalyzes the formation of C16:0-ACP or C18:0-ACP as a component of the FA synthase (FAS) complex [11,53], exhibited a higher expression level in $\mathrm{HO}$ than in LO during seed development (Fig. 10). The KAR gene showed increased expression from 40 to 54 daa, reduced expression at 68 daa, and finally increased expression at the mature stage in the two lines. These results were in accordance with the changes in the relative percentages of palmitic acid (C16:0) and stearic acid (C18:0) of total oils during seed development [54]. Similarly, miR319p_1 was significantly downregulated in $\mathrm{HO}$ compared with $\mathrm{LO}$ at 81 daa (Fig. 8), and expression of its target gene FAD2-2, which desaturates oleic acid (C18:1) to form linoleic acid (C18:2) [11], was slightly increased in $\mathrm{HO}$ at 81 daa (Fig. 10). The expression level of the FAD2-2 gene rapidly increased from 40 to $54 / 68$ daa in the two lines and then decreased at the mature stage (Fig. 10), which correlated well with the changes in linoleic acid and the total FA content in developing yellowhorn seeds [8]. Extensive research suggests that FA biosynthesis in higher plants may be an important regulatory step in oil accumulation $[55,56]$. The enhanced expression of $K A R$ and FAD2-2 genes in the HO line could thereby promote elevated oil accumulation by contributing to an increased FA supply in $\mathrm{HO}$ yellowhorn, revealing that miR7760-p3_1 and miR319p_1 may participate in the regulation of lipid biosynthesis by regulating FA biosynthesis genes $K A R$ and $F A D 2-2$, respectively.

Activated FAs in the form of acyl-CoA are sequentially incorporated into glycerol-3-phosphate to produce triacylglycerols (TAGs) in the TAG synthesis process via the Kennedy pathway. These TAGs are then catalyzed by a series of enzymes, including LPAT and DGAT [57]. In our study, the expression level of LPAT5, targeted by miR1536-p5_2, was higher in HO than in LO during seed development (Fig. 10), while miR1536-p5_2 showed lower expression in $\mathrm{HO}$ compared to $\mathrm{LO}$ at 54, 68, and 81 daa (Fig. 10; Additional file 3: Table S3). Combined with the findings that the $\mathrm{HO}$ line showed higher oil content than the LO line during seed development, it is therefore likely that miR1536-p5_2 mainly played a key regulatory role in lipid biosynthesis in the middle and mature stages of seed development by negatively regulating the TAG biosynthesis gene LPAT5. A rate-limiting reaction in TAG biosynthesis is catalyzed by the DGAT enzyme, and the DGAT1 and DGAT2 genes control the progression of this reaction [10]. Overexpression of AtDGAT1 in seeds increased the seed oil content by up to $8.3 \%$ in transgenic $B$. juncea compared to that in wild-type plants [58]. In yellowhorn, the DGAT1 gene, targeted by miR5647-p3_1, was more highly expressed in the HO line compared to the LO line at 40 daa (Fig. 10), which was in accordance with the difference in the embryo oil content between the two lines. Unlike the DGAT1 gene, miR5647-p3_1 expression was only lower in the HO line at 40 daa (Fig. 10; Additional file 3: Table S3). Thus, it is speculated that miR5647-p3_1 affected primarily seed oil accumulation at the early stage of yellowhorn seed 
development through the suppression of the DGAT1 gene. Although the expression patterns of miR5647p3_1 cannot fully explain the difference in the embryo oil content in the two lines, the possibility of regulation of DGAT1 by miR5647-p3_1 cannot be ruled out at all seed developmental stages. Further molecular genetics studies should be used to determine the role of miR5647-p3_1 in the lipid biosynthesis process.

The Mediator complex plays an essential role in transcriptional regulation in eukaryotes by connecting DNA-binding transcription factors to the RNA polymerase II transcription machinery. It is a large complex comprising 25 to 30 different protein subunits $[59,60]$. MED15 is a subunit of the tail module of the Mediator complex. MED15 includes a kinase-inducible domain-interacting domain at its $\mathrm{N}$-terminal region that mediates protein-protein interactions through a hydrophobic pocket [61]. Several previous studies suggested that MED15 plays an important regulatory role in diverse biological processes, including lipid metabolism, seed development, and defense signaling pathways in many plants $[62,63]$. A recent study found that the Arabidopsis MED15 subunit interacts directly with the WRINKLED1 transcription factor during embryogenesis to mediate the activation of downstream lipidrelated genes. The overexpression of MED15 increased the transcription of glycolysis-related and FA biosynthetic genes, resulting in increased FA content in mature seeds [64]. In yellowhorn, the $M E D 15 A$ gene is targeted by miR7760-p5_1. Interestingly, miR7760-p5_1 showed decreased expression in the HO line compared with the LO line at the mature stage (Fig. 10; Additional file 3: Table S3), which led to higher expression of the MED15A gene in the HO line (Fig. 10). These changes might improve oil accumulation in the $\mathrm{HO}$ line by activating downstream lipid-related genes and further increasing FA biosynthesis. Because a few varieties of FAs and total oil accumulated relatively rapidly between 40 and 68 daa and peaked at the mature stage [8-10], this study focused mainly on activities between 40 and 81 daa, the key period for oil accumulation. Expanding the scope of this work to focus on activities throughout seed development would provide a clearer picture of seed development and the initial stage of oil accumulation.

\section{Conclusions}

In this work, embryos from four seed development stages of two yellowhorn lines ( $\mathrm{HO}$ and $\mathrm{LO}$ ) were used to construct separate small RNA libraries, including two replicates for each sample. A total of 249 known miRNAs belonging to 46 families and 88 novel miRNAs were identified in developing yellowhorn embryos as a result. We screened the differentially expressed miRNAs to obtain miRNAs involved in lipid biosynthesis and seed development regulation by comparing different yellowhorn lines and different developmental stages. The results identified many miRNA-target regulatory modules that have potential functions in regulating seed size, seed weight, and lipid biosynthesis in yellowhorn, including miR172bARF2, miR7760-p3_1-AGL61, miR319p_1-FAD2-2, miR5647-p3_1-DGAT1, and miR7760-p5_1-MED15A. These data will be valuable for dissecting the post-transcriptional and transcriptional regulation of seed development and lipid biosynthesis, as well as improving yellowhorn in northern China.

\section{Methods}

\section{Plant materials and seed phenotype}

Yellowhorn blooms early in May and its fruit ripens in late July. Two yellowhorn lines, NM1203 and NM1003, were grown at the Baxiantong Forest Farm located in Baxiantong Town, Naiman County, Tongliao City, Inner Mongolia Autonomous Region, China (121.04 E, $43.21^{\circ}$ $\mathrm{N})$. The two lines were bred by Dalian Minzu University and established by massal selection of systematic breeding of natural populations. Based on the average yearly embryo oil content, as assessed by bunch analysis over a 3-year period, two yellowhorn lines (NM1203 and NM1003) with similar genetic backgrounds were selected as the HO line and LO line, respectively. The permission was obtained from Dalian Minzu University for the collection of yellowhorn fruits. Fruits were collected at random from different plants of both lines at $40,54,68$, and 81 daa in 2016, which were the same as those for published mRNA-seq data [12]. After collection, the seeds were separated from fruits at the four developmental stages by dissection. The phenotype characters of the seeds were evaluated. These included the hundred-grain weight, transverse diameter, longitudinal diameter, and lateral diameter. The analyses were repeated in triplicate. Liquid nitrogen was used to freeze the fresh embryo tissues and they were stored at $-80^{\circ} \mathrm{C}$ for subsequent use.

\section{Oil content analysis}

The seeds harvested at different times were dried to a constant weight at $80^{\circ} \mathrm{C}$ and then ground using a ball mill. The oil was extracted from the embryos at 40, 54, 68 , and 81 daa as previously described [65]. The experiments were repeated in triplicate.

\section{RNA isolation, small RNA library construction, and sequencing}

Total RNA was extracted using a Trizol reagent (Invitrogen, Carlsbad, CA, USA) from embryo samples of both 
lines following the manufacturer's protocol. A NanoDrop ND-2000 spectrophotometer (Thermo Fischer Scientific, Waltham, MA, USA) was used to assess the purity and quality of RNA. RNA integrity was assessed using the RNA Nano 6000 LabChip Kit of the Agilent Bioanalyzer 2100 system (Agilent Technologies, Santa Clara, CA, USA). sRNA libraries were prepared using the total RNA samples from embryos at each developmental stage from both lines following the protocol of the TruSeq Small RNA Sample Prep Kit (Illumina, San Diego, CA, USA). An Illumina HiSeq2500 Genome analyzer (Illumina) was then used to evaluate and sequence the purified library products at LC Sciences (Hangzhou, China), generating 50-bp single-end reads. Two independent replicates were used for each seed developmental stage from the LO and HO lines.

\section{Primary analysis of the sequencing data sets}

After sequencing, the Illumina pipeline filter (Solexa 0.3) was used to process the raw reads to remove the adaptor dimers, junk reads, and low-complexity sequences, as well as those shorter than $18 \mathrm{nt}$ and longer than $25 \mathrm{nt}$. The resulting clean reads were then mapped onto the Rfam (version 11.0) database (http://rfam.xfam.org/) and Repbase (http://www.girinst.org/) to remove rRNAs, tRNAs, snRNAs, snoRNAs, other Rfam RNAs, and repeat sequences using the Bowtie software (version 1.0.0) [30] in an in-house script ACGT101-miR (version 4.2) (LC Sciences, Houston, Texas, USA) [66], with seed length set to 16 and allowing one mismatch. The remaining sRNA reads after screening were retained for subsequent analysis.

\section{Identification of phasiRNAs and natural antisense siRNAs}

The remaining sRNA reads were mapped to whole genome sequences of yellowhorn [31] using the Bowtie software [30], with seed length set to 16 and allowing one mismatch, to analyze their distribution relative to the reference sequence. The mapped sRNA reads were used to search for phasiRNAs and natural antisense siRNAs (NAT-siRNAs). The sRNA annotation process was conducted as follows: (1) The mapped sRNA reads were used to predict phasiRNAs using the PhaseTank software (version 1.0) [67] with the parameters --island 105 --phasiRNA_abun 50. (2) In the process of NAT-siRNAs identification, NAT gene prediction of the yellowhorn genome was first performed using PlantNATsDB platform [68]. The mapped sRNA reads were further aligned to NAT sequences to identify the sRNA reads originating from NATs using the Bowtie software with the parameters $-\mathrm{v} 1-\mathrm{k} 1$. These sRNA reads originating from NATs were then used to predict clusters (NAT-siRNAs) using the ShortStack software [69] with default parameters.

\section{Identification and prediction of known and novel miRNAs} Known miRNAs were identified by aligning the remaining sRNA reads against the miRBase (version 21.0) database (http://www.mirbase.org) using the Bowtie software [30]. In the alignment, seed length was set to 16 and one mismatch inside the sequence was allowed. The mapped miRNA precursors (hairpins) in the miRBase were further aligned against whole genome sequences of yellowhorn [31] to determine their genomic locations using Megablast software in the in-house script ACGT101-miR, with identity percentage $>90 \%$. Here, known miRNAs were sRNAs that could be aligned to any mature miRNA or miRNA precursor (pre-miRNA) from the plants in miRBase, allowing for up to one mismatch. New 5p- or 3p-derived miRNAs were the sRNAs that matched the other arm of known plant pre-miRNA hairpins opposite the annotated mature miRNA-containing arm. The newly discovered $5 p / 3 p$ sequence was annotated as $p 5 / p 3$, which differs from reported sequences.

After known miRNA prediction, the Bowtie software [30] was used to align remaining unaligned sRNA reads to whole genome sequences of yellowhorn [31], with seed length set to 16 and allowing one mismatch, to identify potentially novel miRNAs. The sRNA flanking genome sequences (120 nt upstream and $120 \mathrm{nt}$ downstream) were extracted and used with RNAfold software (http://rna.tbi.univie.ac.at/cgi-bin/RNAfold. cgi) to predict the RNA secondary structure. The parameters were as follows: (1) number of nucleotides in one bulge of the stem $(<=12)$; (2) number of base pairs in the stem region of the predicted hairpin $(>=16)$; (3) cutoff of free energy $(\mathrm{kcal} / \mathrm{mol}<=-15)$; (4) length of the hairpin (upward and downward stems + terminal loop $>=50)$; (5) length of hairpin loop $(<=200)$; (6) number of nucleotides in one bulge in the mature region $(<=4)$; (7) number of biased errors in one bulge in the mature region $(<=2)$; (8) number of biased bulges in the mature region $(<=2)$; (9) number of errors in the mature region $(<=4)$; (10) number of base pairs in the mature region of the predicted hairpin $(>=12)$; and (11) mature region percentage in the stem $(>=80)$. After alignment analysis, the miRNAs that met the above criteria were considered novel miRNAs.

\section{Differential expression analysis of miRNAs}

The number of clean reads originating from each miRNA represents the expression abundance or level of the corresponding miRNA in small RNA deep sequencing. Normalized read counts were calculated 
for miRNA differential expression analysis according to the following procedures: (1) Find a common set of sequences among all samples. (2) Construct a reference data set. Each data in the reference set is the copy number median value of a corresponding common sequence of all samples. (3) Perform 2-based logarithm transformation on copy numbers $\left(\log _{2}(\operatorname{copy} \#)\right)$ of all samples and reference data set. (4) Calculate the $\log _{2}($ copy\# $)$ difference $\left(\Delta \log _{2}(\right.$ copy\# $\left.)\right)$ between individual sample and the reference data set. (5) Form a subset of sequences by selecting $\mid \Delta \log _{2}($ copy $\#) \mid<2$, which means less than $\left(2^{2}=\right) 4$ fold change from the reference set. (6) Perform linear regressions between individual samples and the reference set on the subset sequences to derive linear equations y $=a_{i} x+b_{i}$, where $a_{i}$ and $b_{i}$ are the slop and interception, respectively, of the derived line, $\mathrm{x}$ is $\log _{2}$ (copy\#) of the reference set, and $y$ is the expected $\log _{2}$ (copy\#) of sample i on a corresponding sequence. (7) Calculate the mid value $x_{\text {mid }}=\frac{\max (\mathrm{x})-\min (\mathrm{x})}{2}$ of the reference set. Calculate the corresponding expected $\log _{2}$ (copy\#) of sample i, $y_{i, \text { mid }}=a_{i} x_{\text {mid }}+b_{i}$. Let $y_{r \text {,mid }}=x_{\text {mid }}$, let $\Delta y_{i}=$ $y_{r, \text { mid }}-y_{i, \text { mid }}$, which is the logarithmic correction factor of sample i. We then derive the arithmetic correction factor $f_{i}=2^{\Delta y i}$ sample i. (8) Correct copy numbers of individual samples by multiplying corresponding arithmetic correction factor fi to original copy numbers. A Student's T-test was used to identify differentially expressed miRNAs between two samples based on normalized read counts. An analysis of variance (ANOVA) was used to identify differentially expressed miRNAs among samples at the four development stages in each line. The miRNAs with $P$-values $<0.05$ were considered differentially expressed.

\section{Target gene predication and enrichment analysis}

The target genes of differentially expressed miRNAs were identified by aligning mature miRNA sequences with published mRNA-seq sequences [12] using TargetFinder (https://github.com/carringtonlab/Targe tFinder) following the procedures described in previous studies $[70,71]$. To study the biological significance of the differentially expressed miRNA targets, GO enrichment analyses were conducted as previously described [12]. GO terms with a $P$-value $<0.05$ were considered significantly enriched. To determine the biochemical metabolic pathways and signal transduction pathways involved by the differentially expressed miRNA targets, KEGG enrichment analyses were performed as those used for GO analysis. Pathways with a $P$-value $<0.05$ were considered significantly enriched by differentially expressed miRNA targets.

\section{Sequence analysis of the target gene}

The target gene encoding Mediator subunit 15a (XsMED15A) was analyzed using the BLAST program and ORF Finder tool at NCBI (http://www.ncbi.nlm.nih.gov/) for the homology study and ORF prediction. The analysis of deduced polypeptides was performed with ExPASy tool (https://www.expasy.org/), which has hyperlinks to different prediction programs, including the ProtParam program for physicochemical properties prediction. Domains, motifs, and other features were identified using the online program SMART (http://smart.embl-heide lberg.de/). The DNAMAN program was used for multiple sequence alignment.

\section{Expression analysis of miRNAs and predicted target genes using qRT-PCR}

The total RNA was isolated from the embryo samples from both lines as described under sRNA library preparation. One microgram of total RNA was used for first-strand cDNA synthesis using PrimeScript RT reagent Kit with gDNA Eraser (TaKaRa, Dalian, China) following the manufacturer's protocol. For miRNA expression analysis, the Mir-X miRNA FirstStrand Synthesis Kit (Takara) was used to perform first-strand cDNA synthesis following the manufacturer's instructions. qRT-PCR was performed using TB Green Premix Ex Taq II (Tli RNaseH Plus) (TaKaRa) on an Applied Biosystems 7500 Real-Time PCR System (Applied Biosystems, Foster City, CA, USA) with the following cycling conditions: $95^{\circ} \mathrm{C}$ for $30 \mathrm{~s}$, followed by 40 cycles of $95^{\circ} \mathrm{C}$ for $5 \mathrm{~s}$ and $60^{\circ} \mathrm{C}$ for $30 \mathrm{~s}$. The miRNA expression-level analysis used yellowhorn 5.8S rRNA as an internal reference, while target expression-level analysis used the $\beta$-actin gene as an internal control. The $2^{-\Delta} \mathrm{CT}$ method was used to calculate the relative expression levels of each miRNA and their targets [72]. The primer sequences used to validate miRNA and target gene expression are listed in Additional file 13: Table S11 and Additional file 14: Table S12, respectively. Three biological replicates were conducted to obtain the data.

\footnotetext{
Abbreviations

miRNA: MicroRNA; sRNA: Small RNA; HO: High-oil-content; LO: Low-oil-content; daa: Days after anthesis; NAT-siRNAs: Natural antisense siRNAs; phasiRNAs: Phased siRNAs; FAs: Fatty acids; DGAT: Diacylglycerol acyltransferase; KAR: 3-oxoacyl-ACP reductase; FAD2: Omega-6 fatty acid desaturase 2; FAE1: Fatty acid elongase 1; FATB: Fatty acyl-ACP thioesterase B; ARF: Auxin response factor; GRF5: Growth-regulating factor 5; ERF3: Ethylene-responsive transcription factor 3; AGL61: AGAMOUS-LIKE 61; WRKY41: WRKY transcription factor 41; LPAT5: Lysophosphatidyl acyltransferase 5; MED15A: Mediator subunit 15a; SDR1: Short-chain type dehydrogenase/reductase; PCMP-H42: Pentatricopeptide repeat-containing protein; FAS: FA synthase; TAG: Triacylglycerol; GO: Gene Ontology; KEGG: Kyoto Encyclopedia of Genes and Genomes; ORF: Open reading frame; PCA: Principal Components Analysis.
} 


\section{Supplementary Information}

The online version contains supplementary material available at https://doi. org/10.1186/s12870-021-03239-4.

Additional file 1: Table S1. Distribution of small RNAs among different categories in $\mathrm{HO}$ yellowhorn.

Additional file 2: Table S2. Distribution of small RNAs among different categories in LO yellowhorn.

Additional file 3: Table S3. The known miRNAs identified in yellowhorn.

Additional file 4: Table S4. Species and abbreviations of the known miRNAs of yellowhorn.

Additional file 5: Table S5. The novel miRNAs identified in yellowhorn.

Additional file 6: Table S6. (a) Differentially expressed miRNAs between $\mathrm{HO} 40$ and HO54. (b) Differentially expressed miRNAs between $\mathrm{HO} 54$ and HO68. (c) Differentially expressed miRNAs between $\mathrm{HO} 68$ and HO81.

Additional file 7: Table S7. (a) Differentially expressed miRNAs between LO40 and LO54. (b) Differentially expressed miRNAs between LO54 and LO68. (c) Differentially expressed miRNAs between LO68 and LO81.

Additional file 8: Table S8. Targets prediction of all differentially expressed miRNAs.

Additional file 9: Table S9. Identified candidate targets for partial known and novel miRNAs.

Additional file 10: Table S10. Enrichment analysis of KEGG pathways of miRNAs and their target genes of yellowhorn.

Additional file 11: Figure S1. Nucleotide and deduced amino acid sequence of CDNA encoding Mediator subunit 15a (XsMED15A). The start codon is marked in red color font. An asterick represents the stop codon.

Additional file 12: Figure S2. Multiple sequence alignment of Mediator subunit 15a from different species. The MED15A protein sequences were chosen from different species and aligned by DNAMAN. The two internal repeats within XsMED15A are shown in black boxes. The predicted coiled coil region is underlined. GenBank accession numbers of MED15A protein sequences used for the comparison are showed as follows: Xanthoceras sorbifolium (Banklt2507240 XsMED15A OK432284), Pistacia vera (XP_031251638.1), Citrus clementina (XP_024036133.1), Juglans regia (XP_035548095.1), Malus domestica (XP_028955279.1), Gossypium hirsutum (XP_040969330.1), Arachis hypogaea (XP_025617476.1), Medicago truncatula (XP_024636817.1), Brassica rapa (XP_033134666.1), and Glycine $\max$ (XP_003547623.1).

Additional file 13: Table S11. Primers for qRT-PCR analysis of differentially expressed miRNAs.

Additional file 14: Table S12. Primers for qRT-PCR analysis of target genes of differentially expressed miRNAs.

\section{Acknowledgements}

Not applicable.

\section{Authors' contributions}

LW and CJR conceived and designed the research; LW and CJR provided funds; LW performed the experiments and analyzed the corresponding results; $A M B$ helped to prepare the materials; CJR and $H L$ helped with the experimental works; LW supervised this whole process and reviewed the manuscript; LW and CJR discussed the results and revised the manuscript. All authors read and approved the final manuscript.

\section{Funding}

This work was supported by the National Natural Science Foundation of China (Grant No. 31901340, 31760213), Key Research and Development Program of Liaoning Province (Grant No. 2020JH2/10200042, 2017204001) and Special Program of Service of National Strategy for Dalian Minzu University (Grant No. 2020fwgj066).

\section{Availability of data and materials}

The datasets generated and analysed during the current study are available in the NCBI Sequence Read Archive (SRA) database under Bioproject PRJNA493982 (https://www.ncbi.nlm.nih.gov/bioproject/?term=PRJNA493982)

\section{Declarations}

\section{Ethics approval and consent to participate}

Authors confrmed that the whole study on plants, including the collection of plant material, complied with institutional, national, and international guidelines and legislation. This study did not require ethical approval or consent, as it did not involve any endangered or protected species.

\section{Consent for publication}

Not applicable.

\section{Competing interests}

The authors declare that they have no competing interests.

\section{Author details}

${ }^{1}$ Key Laboratory of Biotechnology and Bioresources Utilization, Ministry of Education, Institute of Plant Resources, Dalian Minzu University, Dalian 116600, China. ${ }^{2}$ Key Laboratory of Marine Genetics and Breeding (OUC), Ministry of Education, College of Marine Life Science, Ocean University of China, Qingdao 266100, China. ${ }^{3}$ Institute of Economic Forest, Tongliao Academy of Forestry Science and Technology, Tongliao 028000, China.

Received: 1 January 2021 Accepted: 29 September 2021

Published online: 12 October 2021

\section{References}

1. Wang Q, Yang L, Ranjitkar S, Wang JJ, Wang XR, Zhang DX, et al. Distribution and in situ conservation of a relic Chinese oil woody species Xanthoceras sorbifolium (yellowhorn). Can J For Res. 2017;47:1450-6.

2. Yu HY, Fan SQ, Bi QX, Wang SX, Hu XY, Chen MY, et al. Seed morphology, oil content and fatty acid composition variability assessment in yellow horn (Xanthoceras sorbifolium Bunge) germplasm for optimum biodiesel production. Ind Crop Prod. 2017:97:425-30.

3. Venegas-Calerón M, Ruiz-Méndez MV, Martínez Force E, Garcés R, Salas J. Characterization of Xanthoceras sorbifolium Bunge seeds: lipids, proteins and saponins content. Ind Crop Prod. 2017;109:192-8.

4. Ruan CJ, Yan R, Wang BX, Mopper S, Guan WK, Zhang J. The importance of yellow horn (Xanthoceras sorbifolia) for restoration of arid habitats and production of bioactive seed oils. Ecol Eng. 2017;99:504-12.

5. Tang DH, Ruan CJ, Meng T, Ding J. Oil contents and fatty acid composition in different germplasm of Xanthoceras sorbifolia Bunge. China Oils Fats. 2017:42:77-81.

6. Zhang S, Zu YG, Fu YJ, Luo M, Liu W, Li J, et al. Supercritical carbon dioxide extraction of seed oil from yellow horn (Xanthoceras sorbifolia Bunge.) and its anti-oxidant activity. Bioresour Technol. 2010;101:2537-44.

7. Li J, Fu YJ, Qu XJ, Wang W, Luo M, Zhao CJ, et al. Biodiesel production from yellow horn (Xanthoceras sorbifolia Bunge.) seed oil using ion exchange resin as heterogeneous catalyst. Bioresour Technol. 2012;108:112-8.

8. Guo HH, Li QQ, Wang TT, Hu Q, Deng WH, Xia XL, et al. XsFAD2 gene encodes the enzyme responsible for the high linoleic acid content in oil accumulated in Xanthoceras sorbifolia seeds. J Sci Food Agric. 2014:94:482-8.

9. Zhao N, Zhang Y, Li QQ, Li RF, Xia XL, Qin XW, et al. Identification and expression of a stearoyl-ACP desaturase gene responsible for oleic acid accumulation in Xanthoceras sorbifolia seeds. Plant Physiol Biochem. 2015;87:9-16.

10. Guo HH, Wang TT, Li QQ, Zhao N, Zhang Y, Liu D, et al. Two novel diacylglycerol acyltransferase genes from Xanthoceras sorbifolia are responsible for its seed oil content. Gene. 2013;527:266-74.

11. Liu YL, Huang ZD, Ao Y, Li W, Zhang ZX. Transcriptome analysis of yellow horn (Xanthoceras sorbifolia Bunge): a potential oil-rich seed tree for biodiesel in China. PLoS One. 2013;8:e74441. 
12. Wang L, Ruan CJ, Liu LY, Du W, Bao AM. Comparative RNA-Seq analysis of high- and low-oil yellow horn during embryonic development. Int J Mol Sci. 2018;19:3071.

13. Bi QX, Zhao Y, Du W, Lu Y, Gui L, Zheng ZM, et al. Pseudomolecule-level assembly of the Chinese oil tree yellowhorn (Xanthoceras sorbifolium) genome. GigaScience. 2019;8:giz070.

14. Liang Q, Li HY, Li SK, Yuan FL, Sun JF, Duan QC, et al. The genome assembly and annotation of yellowhorn (Xanthoceras sorbifolium Bunge). GigaScience. 2019;8:giz071.

15. Bartel DP. MicroRNAs: target recognition and regulatory functions. Cell. 2009;136:215-33

16. Li SC, Gao FY, Xie KL, Zeng XH, Cao Y, Zeng J, et al. The OsmiR396cOsGRF4-OsGIF1 regulatory module determines grain size and yield in rice. Plant Biotechnol J. 2016:14:2134-46.

17. Li JB, Ding J, Yu X, Li H, Ruan CJ. Identification and expression analysis of critical microRNA-transcription factor regulatory modules related to seed development and oil accumulation in developing Hippophae rhamnoides seeds. Ind Crop Prod. 2019:137:33-42.

18. Nodine MD, Bartel DP. MicroRNAs prevent precocious gene expression and enable pattern formation during plant embryogenesis. Genes Dev. 2010;24:2678-92

19. Zhang H, Zhang JS, Yan J, Gou F, Mao YF, Tang GL, et al. Short tandem target mimic rice lines uncover functions of miRNAs in regulating important agronomic traits. Proc Natl Acad Sci U S A. 2017;114:5277-82.

20. Cao SJ, Zhu QH, Shen WX, Jiao XM, Zhao XC, Wang MB, et al. Comparative profiling of miRNA expression in developing seeds of high linoleic and high oleic safflower (Carthamus tinctorius L.) plants. Front. Plant Sci. 2013;4:489

21. Poudel S, Aryal N, Lu C. Identification of microRNAs and transcript targets in Camelina sativa by deep sequencing and computational methods. PLoS One. 2015;10:e0121542.

22. Chi XY, Yang QL, Chen XP, Wang JY, Pan LJ, Chen MN, et al. Identification and characterization of microRNAs from peanut (Arachis hypogaea L.) by high-throughput sequencing. PLoS One. 2011;6:e27530.

23. Galli V, Guzman F, de Oliveira LFV, Loss-Morais G, Körbes AP, Silva SDA, et al. Identifying microRNAs and transcript targets in Jatropha seeds. PLoS One. 2014;9:e83727.

24. Wang J, Jian HJ, Wang TY, Wei LJ, Li JN, Li C, et al. Identification of microRNAs actively involved in fatty acid biosynthesis in developing Brassica napus seeds using high-throughput sequencing. Front Plant Sci. 2016;7:1570.

25. Belide S, Petrie JR, Shrestha P, Singh SP. Modification of seed oil composition in Arabidopsis by artificial microRNA-mediated gene silencing. Front Plant Sci. 2012;3:168.

26. Na G, Mu X, Grabowski P, Schmutz J, Lu C. Enhancing microRNA167A expression in seed decreases the a-linolenic acid content and increases seed size in Camelina sativa. Plant J. 2019;98:346-58.

27. Ao Y, Wang YW, Chen L, Wang T, Yu HY, Zhang ZX. Identification and comparative profiling of microRNAs in wild-type Xanthoceras sorbifolia and its double flower mutant. Genes Genom. 2012;34:561-8.

28. Ao Y. Characterization and comparison of fower bud microRNAs from yellow-horn species. Genet Mol Res. 2016;15:gmr.15048899.

29. Wang X, Zheng YQ, Su SC, Ao Y. Discovery and profiling of microRNAs at the critical period of sex differentiation in Xanthoceras sorbifolium Bunge. Forests. 2019;10:1141.

30. Langmead B, Trapnell C, Pop M, Salzberg SL. Ultrafast and memoryefficient alignment of short DNA sequences to the human genome. Genome Biol. 2009;10:R25.

31. Bi QX, Zhao Y, Du W, Lu Y, Gui L, Zheng ZM, et al. Supporting data for "Pseudomolecule-level assembly of the Chinese oil tree yellowhorn (Xanthoceras sorbifolium) genome". GigaScience Database. 2019. https:// doi.org/10.5524/100606.

32. Zeng XC, Xu YZ, Jiang JJ, Zhang FQ, Ma L, Wu DW, et al. Identification of cold stress responsive microRNAs in two winter turnip rape (Brassica rapa L.) by high throughput sequencing. BMC Plant Biol. 2018;18:52

33. Huang J, Li ZY, Zhao DZ. Deregulation of the OsmiR160 target gene OsARF18 causes growth and developmental defects with an alteration of auxin signaling in rice. Sci Rep. 2016;6:29938.

34. Shu K, Liu XD, Xie Q, He ZH. Two faces of one seed: hormonal regulation of dormancy and germination. Mol Plant. 2016;9:34-45.
35. Mallory AC, Bartel DP, Bartel B. MicroRNA-directed regulation of Arabidopsis AUXIN RESPONSE FACTOR17 is essential for proper development and modulates expression of early auxin response genes. Plant Cell. 2005;17:1360-75

36. Okushima Y, Mitina I, Quach HL, Theologis A. AUXIN RESPONSE FACTOR 2 (ARF2): a pleiotropic developmental regulator. Plant J. 2005;43:29-46.

37. Schruff MC, Spielman M, Tiwari S, Adams S, Fenby N, Scott RJ. The AUXIN RESPONSE FACTOR 2 gene of Arabidopsis links auxin signalling, cell division, and the size of seeds and other organs. Development. 2006;133:251-61.

38. Chen XM. A microRNA as a translational repressor of APETALA2 in Arabidopsis flower development. Science. 2004;303:2022-5.

39. Jofuku KD, Omidyar PK, Gee Z, Okamuro JK. Control of seed mass and seed yield by the floral homeotic gene APETALA2. Proc Natl Acad Sci U S A. 2005;102:3117-22.

40. Duan PG, Ni S, Wang JM, Zhang BL, Xu R, Wang YX, et al. Regulation of OsGRF4 by OsmiR396 controls grain size and yield in rice. Nat Plants. 2015;2:15203.

41. Sun PY, Zhang WH, Wang YH, He Q, Shu F, Liu H, et al. OsGRF4 controls grain shape, panicle length and seed shattering in rice. J Integr Plant Biol. 2016:58:836-47.

42. Debernardi JM, Mecchia MA, Vercruyssen L, Smaczniak C, Kaufmann K, Inze D, et al. Post-transcriptional control of GRF transcription factors by microRNA miR396 and GIF co-activator affects leaf size and longevity. Plant J. 2014;79:413-26.

43. Horiguchi G, Kim GT, Tsukaya H. The transcription factor AtGRF5 and the transcription coactivator AN3 regulate cell proliferation in leaf primordia of Arabidopsis thaliana. Plant J. 2005;43:68-78.

44. Vercruyssen L, Tognetti VB, Gonzalez N, Van Dingenen J, De Milde L, Bielach A, et al. GROWTH REGULATING FACTOR5 stimulates Arabidopsis chloroplast division, photosynthesis, and leaf longevity. Plant Physiol. 2015;167:817-32

45. Cao DY, Wang J, Ju Z, Liu QQ, Li S, Tian HQ, et al. Regulations on growth and development in tomato cotyledon, flower and fruit via destruction of miR396 with short tandem target mimic. Plant Sci. 2016;247:1-12.

46. Beltramino M, Ercoli MF, Debernardi JM, Goldy C, Rojas AML, Nota F, et al. Robust increase of leaf size by Arabidopsis thaliana GRF3-like transcription factors under different growth conditions. Sci Rep. 2018;8:13447.

47. Debernardi JM, Rodriguez RE, Mecchia MA, Palatnik JF. Functional specialization of the plant miR396 regulatory network through distinct microRNA-target interactions. PLoS Genet. 2012;8:e1002419.

48. Kang IH, Steffen JG, Portereiko MF, Lloyd A, Drews GN. The AGL62 MADS domain protein regulates cellularization during endosperm development in Arabidopsis. Plant Cell. 2008;20:635-47.

49. Chen C, Begcy K, Liu K, Folsom JJ, Wang Z, Zhang C, et al. Heat stress yields a unique MADS box transcription factor in determining seed size and thermal sensitivity. Plant Physiol. 2016;171:606-22.

50. Steffen JG, Kang I-H, Portereiko MF, Lloyd A, Drews GN. AGL61 interacts with AGL80 and is required for central cell development in Arabidopsis. Plant Physiol. 2008;148:259-68.

51. Zhou QY, Zheng YR, Lai LM, Du H. Observations on sexual reproduction in Xanthoceras sorbifolium (Sapindaceae). Acta Bot Occident Sin. 2017:37:0014-22

52. Ye CY, Xu H, Shen EH, Liu Y, Wang Y, Shen YF, et al. Genome-wide identification of non-coding RNAs interacted with microRNAs in soybean. Front Plant Sci. 2014;5:743.

53. Li J, Han DX, Wang DM, Ning K, Jia J, Wei L, et al. Choreography of transcriptomes and lipidomes of nannochloropsis reveals the mechanisms of oil synthesis in microalgae. Plant Cell. 2014;26:1645-65.

54. Liu LY, Ruan CJ, Wang L, Zhang WC, Wang HM, Wu B, et al. Coordinated regulation of multigenes formed by fatty acids in kernel oil of Xanthoceras sorbifolium. Mol Plant Breed. 2019:17:1834-42.

55. Dussert S, Guerin C, Andersson M, Joët T, Tranbarger TJ, Pizot M, et al. Comparative transcriptome analysis of three oil palm fruit and seed tissues that differ in oil content and fatty acid composition. Plant Physiol. 2013;162:1337-58.

56. Troncoso-Ponce MA, Kilaru A, Cao X, Durrett TP, Fan J, Jensen JK, et al. Comparative deep transcriptional profiling of four developing oilseeds. Plant J. 2011;68:1014-27.

57. Coleman RA, Lee DP. Enzymes of triacylglycerol synthesis and their regulation. Prog Lipid Res. 2004;43:134-76. 
58. Savadi S, Naresh V, Kumar V, Bhat SR. Seed-specifc overexpression of Arabidopsis DGAT1 in Indian mustard (Brassica juncea) increases seed oil content and seed weight. Botany. 2016;94:177-84.

59. Malik S, Roeder RG. The metazoan mediator co-activator complex as an integrative hub for transcriptional regulation. Nat Rev Genet. 2010;11:761-72.

60. Bernecky C, Grob P, Ebmeier CC, Nogales E, Taatjes DJ. Molecular architecture of the human mediator-RNA polymerase II-TFIIF assembly. PLOS Biol. 2011;9:e1000603.

61. Parker D, Ferreri K, Nakajima T, LaMorte VJ, Evans R, Koerber SC, et al. Phosphorylation of CREB at Ser-133 induces complex formation with CREBbinding protein via a direct mechanism. Mol Cell Biol. 1996;16:694-703.

62. Canet JV, Dobón A, Tornero P. Non-recognition-of-BTH4, an Arabidopsis mediator subunit homolog, is necessary for development and response to salicylic acid. Plant Cell. 2012;24:4220-35.

63. Thakur JK, Agarwal P, Parida S, Bajaj D, Pasrija R. Sequence and expression analyses of KIX domain proteins suggest their importance in seed development and determination of seed size in rice, and genome stability in Arabidopsis. Mol Gen Genomics. 2013;288:329-46.

64. Kim MJ, Jang IC, Chua NH. The mediator complex MED15 subunit mediates activation of downstream lipid-related genes by Arabidopsis WRINKLED1 transcription factor. Plant Physiol. 2016;171:1951-64.

65. Shockey JM, Gidda SK, Chapital DC, Kuan JC, Dhanoa PK, Bland JM, et al. Tung tree DGAT1 and DGAT2 have nonredundant functions in triacylglycerol biosynthesis and are localized to different subdomains of the endoplasmic reticulum. Plant Cell. 2006;18:2294-313.
66. Han XJ, Yin HF, Song XX, Zhang YX, Liu MY, Sang J, et al. Integration of small RNAs, degradome and transcriptome sequencing in hyperaccumulator Sedum alfredii uncovers a complex regulatory network and provides insights into cadmium phytoremediation. Plant Biotechnol J. 2016;14:1470-83.

67. Guo QL, Qu XF, Jin WB. PhaseTank: genome-wide computational identification of phasiRNAs and their regulatory cascades. Bioinformatics. 2015;31:284-6.

68. Chen DJ, Yuan CH, Zhang J, Zhang Z, Bai L, Meng YJ, et al. PlantNATsDB: a comprehensive database of plant natural antisense transcripts. Nucleic Acids Res. 2012;40:D1187-D93.

69. Axtell MJ. ShortStack: comprehensive annotation and quantification of small RNA genes. RNA. 2013;19:740-51.

70. Allen E, Xie Z, Gustafson AM, Carrington JC. microRNA-directed phasing during trans-acting siRNA biogenesis in plants. Cell. 2005;121:207-21.

71. Schwab R, Palatnik JF, Riester M, Schommer C, Schmid M, Weigel D. Specific effects of microRNAs on the plant transcriptome. Dev Cell. 2005;8:517-27.

72. Schmittgen TD, Livak KJ. Analyzing real-time PCR data by the comparative CT method. Nat Protoc. 2008;3:1101-8.

\section{Publisher's Note}

Springer Nature remains neutral with regard to jurisdictional claims in published maps and institutional affiliations.
Ready to submit your research? Choose BMC and benefit from:

- fast, convenient online submission

- thorough peer review by experienced researchers in your field

- rapid publication on acceptance

- support for research data, including large and complex data types

- gold Open Access which fosters wider collaboration and increased citations

- maximum visibility for your research: over $100 \mathrm{M}$ website views per year

At BMC, research is always in progress.

Learn more biomedcentral.com/submissions 